\title{
Observing a changing Hilbert-space inner product
}

\author{
Salini Karuvade $\odot$, Abhijeet Alase $\odot$, and Barry C. Sanders $\odot$ \\ Institute for Quantum Science and Technology, University of Calgary, 2500 University Drive NW, Calgary, Alberta T2N 1N4, Canada
}

(Received 1 January 2021; accepted 16 December 2021; published 7 January 2022)

\begin{abstract}
In quantum mechanics, physical states are represented by rays in Hilbert space $\mathscr{H}$, which is a vector space imbued by an inner product $\langle\mid\rangle$, whose physical meaning arises as the overlap $\langle\phi \mid \psi\rangle$ for $|\psi\rangle$ a pure state (description of preparation) and $\langle\phi|$ a projective measurement. However, current quantum theory does not formally address the consequences of a changing inner product during the interval between preparation and measurement. We establish a theoretical framework for such a changing inner product, which we show is consistent with standard quantum mechanics. Furthermore, we show that this change is described by a quantum operation, which is tomographically observable, and we elucidate how our result is strongly related to the exploding topic of PT-symmetric quantum mechanics. We explain how to realize experimentally a changing inner product for a qubit in terms of a qutrit protocol with a unitary channel.
\end{abstract}

DOI: 10.1103/PhysRevResearch.4.013016

Hilbert-space inner product is fundamental to quantum mechanics $(\mathrm{QM})$, and its physicality relates to norm through the Born interpretation and to fidelity and distinguishability through its complex angle [1]. The uniqueness of the inner product associated to a quantum system has come under scrutiny following the advent of PT-symmetric QM. PT-symmetric systems are described by non-Hermitian Hamiltonians invariant under the combined action of parity $(\mathrm{P})$ and time (T) inversion symmetries [2-5], and they are predicted to exhibit novel physical phenomena, which have been simulated on a variety of experimental platforms [6-14]. These phenomena have been explained by observing that non-Hermitian Hamiltonians with unbroken PT symmetry are Hermitian with respect to a different Hilbert-space inner product [3,15-17]. Changing Hilbert-space inner product is valuable for certain quantum information processing (QIP) tasks [18] such as nonorthogonal state discrimination [19], cloning [20], and quantum algorithms [21,22], but perfunctory applications have led to counter-factual conclusions $[18,23,24]$ including violation of the no-signalling principle [25]. Our aim is to prescribe the correct procedure for changing Hilbert-space inner product and to devise an experiment to validate our prescription.

Consistency of a changing Hilbert-space inner product with standard QM and the unobservability of such a change in closed systems have been investigated. A $\mathrm{C}^{*}$-algebraic approach shows that a set of non-Hermitian operators comprises the observables of a quantum mechanical system if and only if the operators are Hermitian with respect to a new Hilbertspace inner product [26]. Such a modified inner product is

Published by the American Physical Society under the terms of the Creative Commons Attribution 4.0 International license. Further distribution of this work must maintain attribution to the author(s) and the published article's title, journal citation, and DOI. the key to proving the equivalence of PT-symmetric QM with the Dirac-von Neumann formulation of QM in the case of closed systems, i.e., systems in which every time evolution is a unitary operation [3,15,17,27-31]. Furthermore, this equivalence implies that any change in inner product is unobservable in experiments on closed systems [32]. Therefore, the above proposals that use the inner-product change for QIP tasks as well as the counter-factual claims are not applicable to closed systems.

Evolution generated by PT-symmetric Hamiltonians has been implemented experimentally for applications including sensing [33-35], cloaking [36,37], and unidirectional propagation [38]. These experiments simulate PT-symmetric dynamics on classical $[6,7,9,10]$ or quantum $[8,11,39,40]$ systems by balancing loss and gain. Another way to simulate PT-symmetric Hamiltonians with real spectra is by dilating the nonunitary propagator to a nonlocal unitary operator over multiple subsystems, which has been demonstrated on qubit systems [13,41-48]. However, none of these simulation strategies involve effecting a change of inner product.

PT-symmetric Hamiltonians and a changing Hilbert-space inner product are known to be consistent with standard QM for closed systems, but they are not yet known to be consistent for open systems. To solve these outstanding problems, we construct an operational framework, consistent with the $\mathrm{C}^{*}$ algebraic formulation of $\mathrm{QM}$, which accommodates a change in inner product between preparation and measurement. Furthermore, neither PT symmetry nor a changing Hilbert-space inner product are observable in closed systems, but could be observable in open systems [32]. We show our change in inner product is implemented by a quantum operation (henceforth assumed to be completely positive and trace nonincreasing), which can be observed using tomography. Next we connect our framework to the burgeoning topic of PT-symmetric QM by explaining how an inner-product-changing quantum operation can be used to implement PT-symmetric dynamics in an open system. Finally, at the empirical level, we describe a 
(a)

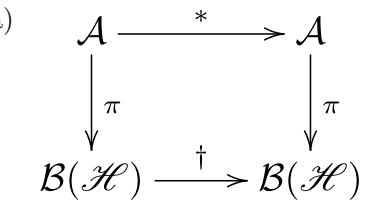

(b)

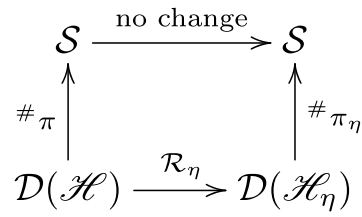

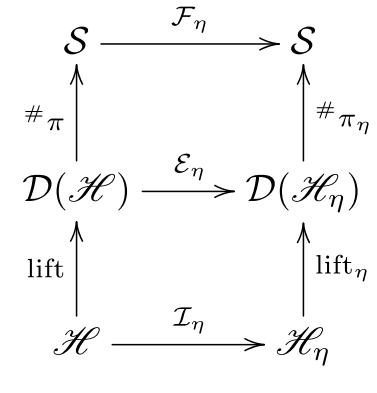

FIG. 1. (a) Diagram illustrating the relation between the * operation of $\mathcal{A}$ and the $\dagger$ operation of $\mathcal{B}(\mathscr{H})$ under the representation $\pi$. (b) Commutative diagram depicting the change of representation from $\pi$ to $\pi_{\eta}$ under $\mathcal{R}_{\eta}$. Operationally, $\mathcal{R}_{\eta}$ represents a trivial transformation, i.e., no change, in $S$. (c) Commutative diagram illustrating the relation between the maps $\mathcal{F}_{\eta}, \mathcal{E}_{\eta}$, and $\mathcal{I}_{\eta}$.

potential experimental simulation for changing the inner product of a qubit by subjecting a qutrit to unitary evolution and neglecting the third Hilbert-space dimension during preparation and measurement but not during evolution. We also extend this simulation procedure to $d$-dimensional systems.

To construct the operational framework for changing the inner product associated to a quantum system between preparation and measurement, we adopt the $\mathrm{C}^{*}$-algebraic framework of QM [49], which provides freedom in representing a given system on different Hilbert spaces following the Gel'fand-Naimark-Segal (GNS) construction [50,51]. We employ this representation freedom first to construct representations of the $C^{*}$ algebra on a pair of Hilbert spaces whose inner products are related by a given metric operator $\eta$. We then define the change in inner product by $\eta$ as the identity isomorphism between the two Hilbert spaces. To operationalize the change in inner product, we use commutative diagrams that connect this isomorphism to a quantum operation between the bounded operators on the two Hilbert spaces and finally observe that the quantum operation induces an observable physical transformation on the system.

In the operational approach, the operators of a quantum system form a unital $\mathrm{C}^{*}$ algebra $\mathcal{A}=\{A\}$, which is equipped with $\mathrm{a} *$ operation that captures the notion of adjoint. The algebra $\mathcal{A}$ is representable on a possibly infinite dimensional Hilbert space $\mathscr{H}=(\mathscr{V},\langle\mid\rangle)$, comprising a complete vector space $\mathscr{V}$ and an inner product $\langle\mid\rangle$, which is a nondegenerate sesquilinear form. In Fig. 1(a), observables are self-adjoint elements of $\mathcal{A}$ and correspond to allowed measurements. A representation of $\mathcal{A}$ is a product-preserving linear map

$$
\mathcal{A} \stackrel{\pi}{\rightarrow} \mathcal{B}(\mathscr{H}): \pi\left(A^{*}\right)=(\pi(A))^{\dagger},
$$

where $\mathcal{B}(\mathscr{H})$ denotes the space of bounded linear operators acting on $\mathscr{H}$ and ${ }^{\dagger}$ denotes the Hermitian conjugate. Such a representation can be obtained using the GNS construction [50,51]. Product preservation ensures that if $I$ is the identity operator in $\mathcal{A}$, then $\pi(I)$ is the identity operator in $\mathcal{B}(\mathscr{H})$. An operator $M \in \mathcal{B}(\mathscr{H})$ satisfying $M^{\dagger}=M$ is called a selfadjoint or a Hermitian operator.

We now define states and explain how to represent states as operators on Hilbert space. States correspond to allowed

preparations of the system [Fig. 1(b)]. A state $\omega$ is a positive linear functional on $\mathcal{A}$ that is normalized, i.e., $\omega(I)=1$. This definition is extended to include any subnormalized positive linear functional, i.e., $\omega(I) \leqslant 1$, which corresponds to probabilistic preparation in the state $\omega / \omega(I)$ with probability $\omega(I)$ [52]. Supernormalized positive linear functionals are not valid states according to this probabilistic interpretation [53]. Let $\mathcal{D}(\mathscr{H}):=\left\{\rho: \rho \geqslant 0, \rho=\rho^{\dagger}, \operatorname{tr}(\rho) \leqslant 1\right\} \subset \mathcal{B}(\mathscr{H})$ denote the set of density operators with $\rho \in \mathcal{D}(\mathscr{H})$ representing a state $\omega$ by $\rho \stackrel{{ }_{\pi}}{\mapsto} \omega$ if and only if the expectation value $\operatorname{tr}(\rho \pi(A))=\omega(A) \forall A$. As ${ }^{\#} \pi$ is uniquely determined by $\pi$, we say that $\omega$ is represented by $\rho \in \mathcal{D}(\mathscr{H})$ under $\pi$. We denote by $\mathcal{S}=\{\omega\}$, the set of all states that are represented by density operators under $\pi$. For the special case of pure $\omega$, $\rho=|\psi\rangle\langle\psi|$ for some $|\psi\rangle \in \mathscr{H}$ with $\langle\psi \mid \psi\rangle \leqslant 1$. The transformation $|\psi\rangle \stackrel{\text { lift }}{\mapsto}|\psi\rangle\langle\psi|$ relates Hilbert-space vectors to the density operators in $\mathcal{D}(\mathscr{H})$ [54].

Now that we have explained states and their representations, we now discuss changing representation to being over a different Hilbert space. Given a self-adjoint positivedefinite metric operator $\eta \in \mathcal{B}(\mathscr{H})$, a new Hilbert space $\mathscr{H}_{\eta}=\left(\mathscr{V},\langle\mid\rangle_{\eta}\right)$ can be constructed [26] such that the inner products of the two Hilbert spaces are related by $\langle\bullet \mid \bullet\rangle_{\eta}:=$ $\langle\bullet \mid \eta \bullet\rangle$. A representation $\pi_{\eta}$ on $\mathscr{H}_{\eta}$ can be constructed through (see Appendix A)

$$
\pi(\bullet) \stackrel{\mathcal{R}_{\eta}}{\mapsto} \pi_{\eta}(\bullet):=R \pi(\bullet) R^{-1}, \mathcal{R}_{\eta}: \mathcal{B}(\mathscr{H}) \rightarrow \mathcal{B}\left(\mathscr{H}_{\eta}\right),
$$

where $R=\eta^{-1 / 2}$ is a linear isometry from $\mathscr{H}$ to $\mathscr{H}_{\eta}$. We note that this isometry has been used to prove that Hamiltonians with unbroken PT symmetry are consistent with standard QM, in the case of closed systems [18,27-29,41]. We refer to the quantum channel $\mathcal{R}_{\eta}$ as a "change in representation" [Fig. 1(b)]. In representation $\pi_{\eta}$, the state $\omega \stackrel{\pi^{\pi_{\eta}}}{\longleftrightarrow} \mathcal{R}_{\eta}(\rho)$ such that $\operatorname{tr}\left(\mathcal{R}_{\eta}(\rho) \pi_{\eta}(A)\right)=\omega(A) \forall A$, and $\mathcal{R}_{\eta}(\rho) \neq \rho$ in general.

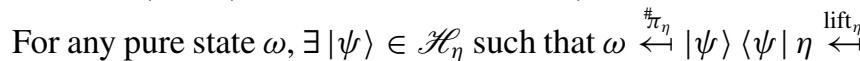
$|\psi\rangle$ (see Appendix A3). As

$$
\operatorname{tr}(\rho \pi(A))=\operatorname{tr}\left(\mathcal{R}_{\eta}(\rho) \pi_{\eta}(A)\right) \forall \omega, A,
$$

representations $\pi$ and $\pi_{\eta}$ are physically, i.e., observationally, indistinguishable. The right-hand side of Eq. (3) can also be interpreted as preparation (state) described in $\pi$ followed by a change in representation from $\pi$ to $\pi_{\eta}$ effected by $\mathcal{R}_{\eta}$ and finally measurement (observable) described in $\pi_{\eta}$. Change in representation between preparation and measurement sets the stage for our definition of change in inner product.

We define a change in inner product by $\eta$ to be the identity isomorphism $\mathcal{I}_{\eta}: \mathscr{H} \rightarrow \mathscr{H}_{\eta}$ such that every $|\psi\rangle \mapsto|\psi\rangle$. For any pair $|\psi\rangle,|\phi\rangle \in \mathscr{H}$, the inner product between the pair of transformed vectors $\mathcal{I}_{\eta}|\psi\rangle, \mathcal{I}_{\eta}|\phi\rangle$ is $\langle\psi|\eta| \phi\rangle$, and the change is trivial if $\eta=\pi(I)$; i.e., for all pairs $|\psi\rangle,|\phi\rangle$, $\langle\psi|\eta| \phi\rangle=\langle\psi \mid \phi\rangle$. Our definition is motivated by proposals to effect PT-symmetric evolution and measurement by changing the Hilbert-space inner product $[19,21]$ but without a prescription for making such changes operationally or mathematically. Next we explain separately, for the cases $\eta \leqslant \pi(I)$ and $\eta \not \leq$ $\pi(I)$, how the isomorphism $\mathcal{I}_{\eta}$ can be physically realized as a quantum operation. 


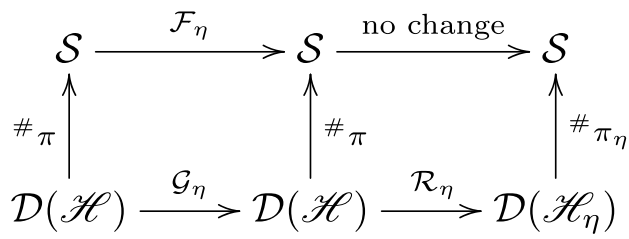

FIG. 2. Commutative diagram showing the action of $\mathcal{F}_{\eta}$ decomposed in terms of $\mathcal{G}_{\eta}$ and $\mathcal{R}_{\eta}$.

The change in inner product by $\eta \leqslant \pi(I)$ is physically realizable via the operation

$$
\mathcal{E}_{\eta}: \mathcal{B}(\mathscr{H}) \rightarrow \mathcal{B}(\mathscr{H} \eta): M \mapsto M \eta,
$$

which is not trace preserving for $\eta \neq \pi(I)$ (see Appendix B). The operation $\mathcal{E}_{\eta}$ mimics the action of $\mathcal{I}_{\eta}$ at the level of density operators, because $|\psi\rangle \stackrel{\text { lift }}{\mapsto}|\psi\rangle\langle\psi|$ whereas $\mathcal{I}_{\eta}|\psi\rangle \stackrel{\text { lift }}{\mapsto}$ $|\psi\rangle\langle\psi| \eta=\mathcal{E}_{\eta}(|\psi\rangle\langle\psi|)$. The operation $\mathcal{E}_{\eta}$ induces a linear map $\mathcal{F}_{\eta}: \mathcal{S} \rightarrow \mathcal{S}$ such that, for any pure $\omega \in \mathcal{S}$, both $\omega$ and $\mathcal{F}_{\eta}(\omega)$ are represented by the same $|\psi\rangle$ under the representations $\pi$ and $\pi_{\eta}$ respectively. However, $\omega$ and $\mathcal{F}_{\eta}(\omega)$ are not necessarily the same state [Fig. 1(c)]. Even in the special case where the two states differ by a scaling factor, they are inequivalent in our setting. The expectation value of $I$ with respect to $\mathcal{F}_{\eta}(\omega)$ gives the success probability of the innerproduct changing quantum operation on the state $\omega . \mathcal{E}_{\eta}$ can be implemented experimentally by lossy purity-preserving operations, i.e., operations that are not necessarily deterministic and transform the set of pure states into itself. In the Heisenberg picture, the operators transform according to the map

$$
\mathcal{E}_{\eta}^{\text {op }}: \mathcal{B}(\mathscr{H}) \rightarrow \mathcal{B}\left(\mathscr{H}_{\eta}\right): M \mapsto \eta M .
$$

This transformation $\mathcal{E}_{\eta}^{\text {op }}$ could modify commutator relations as we show in Appendix $\mathrm{C}$.

In the case $\eta \not \leq \pi(I), \mathcal{E}_{\eta}$ is completely positive but traceincreasing for some $\rho \in \mathcal{B}(\mathscr{H})$ and hence not a quantum operation. In such cases, a scaled version of change in inner product can be implemented in the following way: choose $\kappa \in(0,1)$ such that $\kappa \eta \leqslant \pi(I)$ and observe that $\mathcal{E}_{\kappa \eta}=\kappa \mathcal{E}_{\eta}$ with $\mathcal{E}_{\kappa \eta}$ a quantum operation. Therefore, $\mathcal{E}_{\kappa \eta}$ implements change in inner product by $\eta \not \leq \pi(I)$ up to a scaling factor $\kappa$. Such a scaled version of change in inner product is useful to reverse the effect of operation $\mathcal{E}_{\eta}$ when $\eta \leqslant \pi(I)$. In this case, the isomorphism $\mathcal{I}_{\eta^{-1}}: \mathscr{H}_{\eta} \rightarrow \mathscr{H}$ reverses the change in inner product and the corresponding $\mathcal{E}_{\eta^{-1}}$ is not a valid operation because $\eta^{-1} \geqslant \pi_{\eta}(I)$. Nevertheless, we can choose $\kappa=1 /\left\|\eta^{-1}\right\|$, where $\|\bullet\|$ denotes the operator norm [55], and observe that $\mathcal{E}_{\kappa \eta^{-1}} \circ \mathcal{E}_{\eta}(\rho)=\kappa \rho$ for all $\rho \in \mathcal{B}(\mathscr{H})$. Therefore, the operation $\mathcal{E}_{\kappa \eta^{-1}}: \mathcal{B}\left(\mathscr{H}_{\eta}\right) \rightarrow \mathcal{B}(\mathscr{H})$ reverses, with probability $\kappa$, the change in inner product by $\eta$.

The metric operator $\eta$ can be estimated via quantum process tomography [56] for $\eta \leqslant \pi(I)$, or $\kappa \eta$ if otherwise. The change in inner product by $\eta \leqslant \pi(I)$ is implemented via the operation (Fig. 2)

$$
\mathcal{E}_{\eta}=\mathcal{R}_{\eta} \circ \mathcal{G}_{\eta}, \mathcal{G}_{\eta}: \mathcal{B}(\mathscr{H}) \rightarrow \mathcal{B}(\mathscr{H}): M \mapsto \eta^{1 / 2} M \eta^{1 / 2},
$$

for the Kraus rank-1 operation $\mathcal{G}_{\eta}$. Then the Kraus operator $\eta^{1 / 2}$ and therefore $\eta$ can be estimated by quantum process tomography for trace nonincreasing channels [57]. In the other

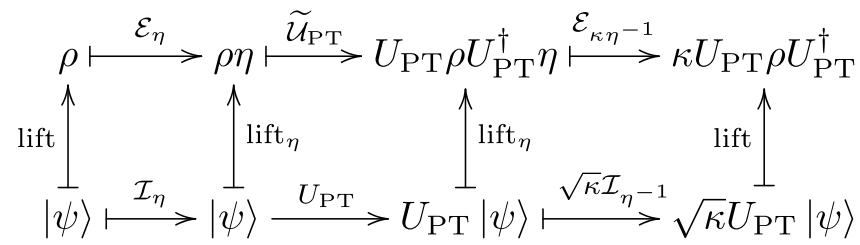

FIG. 3. Diagram showing implementation of a PT-symmetric dynamics using change in inner product and unitary dynamics.

case $\eta \not \leq \pi(I)$, the change in inner product is implemented by the operation $\mathcal{E}_{\kappa \eta}$ from which $\kappa \eta$ is estimated similarly; however, the above procedure does not yield $\kappa$ and $\eta$ separately.

We now discuss how to implement dynamics generated by a diagonalizable Hamiltonian $H_{\mathrm{PT}}$ with unbroken PT symmetry in finite dimensions over a time $t \geqslant 0$, by building on our framework for changing inner product. The dynamical transformation generated by $H_{\mathrm{PT}}$ is

$$
\rho \stackrel{\mathcal{U}_{\mathrm{PT}}}{\mapsto} \kappa U_{\mathrm{PT}} \rho U_{\mathrm{PT}}^{\dagger}, U_{\mathrm{PT}}:=\mathrm{e}^{-\mathrm{i} H_{\mathrm{PT}} t / \hbar},
$$

for some $\kappa \in(0,1)$, where both $\rho, \mathcal{U}_{\mathrm{PT}}(\rho) \in \mathcal{D}(\mathscr{H})$ represent states under $\pi$. We show that this dynamics can be implemented by first changing the inner product, then applying an appropriate unitary channel and finally reversing the change in inner product. To explain this sequence, we consider an arbitrary pure state represented by $|\psi\rangle \in \mathscr{H}$, which is to be transformed to that represented by $\sqrt{\kappa} U_{\mathrm{PT}}|\psi\rangle \in \mathscr{H}$ (lower row of Fig. 3). Next, we compute a metric operator $\eta \leqslant$ $\pi(I) \in \mathcal{B}(\mathscr{H})$ that satisfies the quasi-Hermiticity condition

$$
H_{\mathrm{PT}}^{\dagger}=\eta H_{\mathrm{PT}} \eta^{-1}
$$

the existence of such an $\eta$ is guaranteed as $H_{\mathrm{PT}}$ has unbroken PT symmetry [58]. The Hamiltonian $H_{\mathrm{PT}}$ is self-adjoint with respect to the inner product of the new Hilbert space $\mathscr{H}_{\eta}$. Therefore, $U_{\text {PT }}$ represents unitary dynamics on $\mathscr{H}_{\eta}$, which constitutes the second step of the sequence. Prior to implementing $U_{\mathrm{PT}}$, we transform $|\psi\rangle \in \mathscr{H}$ to $|\psi\rangle \in \mathscr{H}_{\eta}$ via a change in inner product using $\mathcal{I}_{\eta}$. Finally, the transformation from $U_{\mathrm{PT}}|\psi\rangle \in \mathscr{H}_{\eta}$ to $\sqrt{\kappa} U_{\mathrm{PT}}|\psi\rangle \in \mathscr{H}$ is equivalent to reversing the change in inner product using $\mathcal{I}_{\eta^{-1}}$ with probability $\kappa$. This sequence extends to general mixed states by the application of lift, lift $_{\eta}$ maps and linearity (upper row of Fig. 3); here $\tilde{\mathcal{U}}_{\mathrm{PT}} \in \mathcal{B}\left(\mathscr{H}_{\eta}\right)$ is the unitary channel satisfying $\operatorname{lift}_{\eta}\left(U_{\mathrm{PT}}|\psi\rangle\right)=\widetilde{\mathcal{U}}_{\mathrm{PT}}\left(\operatorname{lift}_{\eta}(|\psi\rangle)\right)$, for all $|\psi\rangle \in \mathscr{H}_{\eta}$.

PT-symmetric dynamics in Eq. (7) can be expressed as a sequence of channels acting exclusively on $\mathcal{B}(\mathscr{H})$, thereby paving the way for experimental simulation of PT-symmetric systems. Following the upper row of Fig. 3 , we start by expressing $\mathcal{U}_{\mathrm{PT}}$ [Eq. (7)] as $\mathcal{U}_{\mathrm{PT}}=\mathcal{E}_{\kappa \eta^{-1}} \circ \tilde{\mathcal{U}}_{\mathrm{PT}} \circ \mathcal{E}_{\eta}$. Similar to Eq. (6), we express the reverse change in inner product as $\mathcal{E}_{\kappa \eta^{-1}}=\mathcal{G}_{\kappa \eta^{-1}} \circ \mathcal{R}_{\kappa \eta^{-1}}$, for $\mathcal{G}_{\kappa \eta^{-1}}: \mathcal{B}(\mathscr{H}) \rightarrow \mathcal{B}(\mathscr{H})$ : $\mathcal{G}_{\kappa \eta^{-1}}(M)=\kappa \eta^{-1 / 2} M \eta^{1 / 2}$ and $\mathcal{R}_{\kappa \eta^{-1}}: \mathcal{B}(\mathscr{H} \eta) \rightarrow \mathcal{B}(\mathscr{H})$ is the channel effecting the change in representation form $\pi_{\eta}$ to $\pi$. We then rewrite $\mathcal{U}_{\mathrm{PT}}$ as

$$
\mathcal{U}_{\mathrm{PT}}=\mathcal{G}_{\kappa \eta^{-1}} \circ\left(\mathcal{R}_{\kappa \eta^{-1}} \circ \tilde{\mathcal{U}}_{\mathrm{PT}} \circ \mathcal{R}_{\eta}\right) \circ \mathcal{G}_{\eta},
$$

which is the desired decomposition. The channels $\mathcal{G}_{\kappa \eta^{-1}}, \mathcal{G}_{\eta}$ have single Kraus operators $\sqrt{\kappa} \eta^{-1 / 2}$ and $\eta^{1 / 2}$ respectively. 


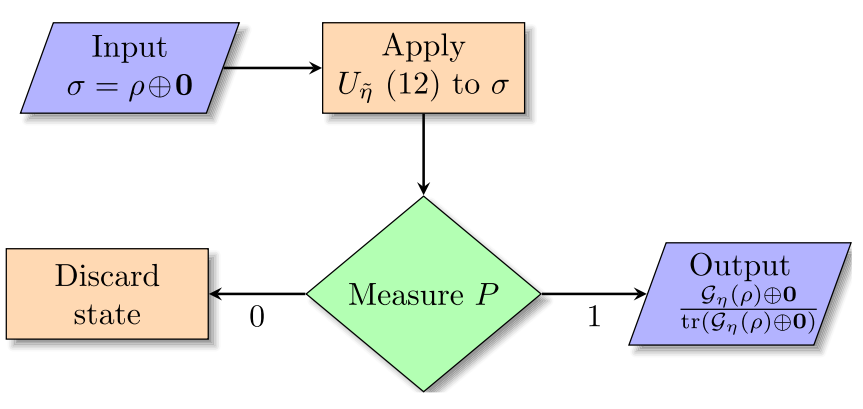

FIG. 4. Simulation of the application of $\mathcal{G}_{\eta}$ on $\rho$ with success probability $\operatorname{tr}\left(\mathcal{G}_{\tilde{\eta}}(\rho) \oplus \mathbf{0}\right)$.

The maps $\mathcal{R}_{\eta}, \mathcal{R}_{\kappa \eta^{-1}}$ only effect change in representation and operationally are equivalent to no change. Finally, the transformation $\mathcal{R}_{\kappa \eta^{-1}} \circ \mathcal{U}_{\mathrm{PT}} \circ \mathcal{R}_{\eta}$ implements a channel corresponding to the unitary Kraus-operator $\eta^{1 / 2} U_{\mathrm{PT}} \eta^{-1 / 2}$ acting on $\mathscr{H}$, generated by the Hamiltonian

$$
h_{\mathrm{PT}}=\eta^{1 / 2} H_{\mathrm{PT}} \eta^{-1 / 2} \in \mathcal{B}(\mathscr{H}) \text {, }
$$

which can be verified to be self-adjoint, i.e., $h_{\mathrm{PT}}^{\dagger}=h_{\mathrm{PT}}$, using the quasi-Hermiticity condition in Eq. (8).

We now design a qutrit procedure for an agent to simulate successfully the change in inner product by $\eta \leqslant \pi(I)$ of a qubit system with algebra $\mathcal{A}$, which is represented on a twodimensional Hilbert space $\mathscr{H}_{2}$ by $\pi$. Our procedure, which shall simulate the operation $\mathcal{G}_{\eta}$ (Fig. 2), uses a unitary operation on the three-dimensional Hilbert space $\mathscr{H}_{3}=\mathscr{H}_{2} \oplus \mathscr{H}_{1}$ followed by a projective measurement on to $\mathscr{H}_{2}$ and postselection, as we now explain. For any $\eta \leqslant \pi(I)$, we first construct the metric operator

$$
\tilde{\eta}:=\frac{1}{\|\eta\|} \eta \Rightarrow \mathcal{G}_{\eta}=\|\eta\| \mathcal{G}_{\tilde{\eta}},
$$

and the unitary operator $U_{\tilde{\eta}} \in \mathcal{B}\left(\mathscr{H}_{3}\right)$ that satisfies

$$
\mathcal{G}_{\tilde{\eta}}(\rho) \oplus \mathbf{0}=P U_{\tilde{\eta}} \sigma U_{\tilde{\eta}}^{\dagger} P, \sigma:=\rho \oplus \mathbf{0}, \forall \rho \in \mathcal{B}\left(\mathscr{H}_{2}\right),
$$

where $P$ is the orthogonal projector on $\mathscr{H}_{2}$. The matrix representation of $U_{\tilde{\eta}}$ is (see Appendix D)

$$
\left[U_{\tilde{\eta}}\right]=\left(\begin{array}{cc}
{[\tilde{\eta}]^{\frac{1}{2}}} & \boldsymbol{u} \\
-\mathrm{e}^{\mathrm{i} \theta} \overline{\boldsymbol{u}}^{\top} & \mathrm{e}^{\mathrm{i} \theta} r
\end{array}\right), \operatorname{spec}\left(\tilde{\eta}^{1 / 2}\right)=\{1, r\}, \theta \in[0,2 \pi),
$$

where [ ] denotes matrix representation, $\boldsymbol{u}$ is the eigenvector of $[\tilde{\eta}]^{1 / 2}$ with eigenvalue $r$ and $\|\boldsymbol{u}\|=\sqrt{1-r^{2}}$. Furthermore, $\overline{\boldsymbol{u}}^{\top}$ is the Hermitian conjugate of the vector $\boldsymbol{u}$. Both $\theta$ and the global phase of $\boldsymbol{u}$ are free parameters. The qutrit unitary operator $U_{\tilde{\eta}}$ is part of the overall simulation procedure [Eq. (12)].

Now we explain how an agent can sequentially apply each operator in Eq. (12) to simulate $\mathcal{G}_{\eta}$ (Fig. 4). The agent is provided with a description of $2 \times 2$ matrix $[\eta]$, in the logical basis $\{|0\rangle,|1\rangle\}$ and a quantum state $\sigma$ [Eq. (12)]. The task is to generate the state $\left(\mathcal{G}_{\eta}(\rho) \oplus \mathbf{0}\right) / \operatorname{tr}\left(\mathcal{G}_{\eta}(\rho) \oplus \mathbf{0}\right)$ with probability $\operatorname{tr}\left(\mathcal{G}_{\tilde{\eta}}(\rho) \oplus \mathbf{0}\right)$. The agent first computes [ $\left.\tilde{\eta}\right]$ [Eq. (11)] and $\left[U_{\tilde{\eta}}\right]$ [Eq. (13)] and then applies physical operations corresponding to $U_{\tilde{\eta}}$ on $\sigma$ followed by projective measurement $P$ [Eq. (12)]. For nonzero measurement outcome, which occurs with probability $\operatorname{tr}\left(\mathcal{G}_{\tilde{\eta}}(\rho) \oplus \mathbf{0}\right)$, the postmeasurement state ob- tained is [Eq. (11)]

$$
\frac{\mathcal{G}_{\tilde{\eta}}(\rho) \oplus \mathbf{0}}{\operatorname{tr}\left(\mathcal{G}_{\tilde{\eta}}(\rho) \oplus \mathbf{0}\right)}=\frac{\mathcal{G}_{\eta}(\rho) \oplus \mathbf{0}}{\operatorname{tr}\left(\mathcal{G}_{\eta}(\rho) \oplus \mathbf{0}\right)} .
$$

The agent discards the state if the measurement outcome is zero. This concludes the simulation procedure. The agent may further estimate the success probability $\operatorname{tr}\left(\mathcal{G}_{\tilde{\eta}}(\rho) \oplus \mathbf{0}\right)$, if required, by repeating the simulation procedure on a large number of copies of $\sigma$ provided to them and then calculating the ratio of nonzero measurement outcomes to the total number of copies used [59].

In Appendix E, we provide an explicit procedure to simulate the dynamics [Eq. (7)] of the qubit PT-symmetric Hamiltonian [2], by sequentially applying the operators in Eq. (9) and by using the qutrit simulation procedure to implement $\mathcal{G}_{\eta}, \mathcal{G}_{\kappa \eta^{-1}}$. In Appendix F, we design a simulation procedure, similar to our qutrit procedure given above, for changing the inner product of a $d$-dimensional system using a $2 d$-dimensional system for any positive integer $d$. Furthermore, we use our procedure to simulate the dynamics of a $d$-dimensional PT-symmetric Hamiltonian by using only $2 d$ dimensions, instead of $d^{3}$ dimensions as required in the Stinespring dilation approach [60].

We also design a scheme to verify tomographically whether a prover can perform an arbitrary change of inner product using our qutrit simulation procedure. Input to the verification scheme is a threshold function $D_{\text {th }}: \mathcal{B}\left(\mathscr{H}_{2}\right) \rightarrow(0,1)$ given as a black-box. The output is "accept" if $\| \mathcal{G}_{\eta} \oplus \mathbf{0}-$ $\hat{\mathcal{G}}_{\eta} \|_{1 \rightarrow 1} \leqslant D_{\text {th }}(\eta)$ or "reject" otherwise, where $\hat{\mathcal{G}}_{\eta}: \mathcal{B}\left(\mathscr{H}_{3}\right) \rightarrow$ $\mathcal{B}\left(\mathscr{H}_{3}\right)$ represents a tomographic reconstruction of the qutrit process implemented by the prover, $\mathcal{G}_{\eta} \oplus \mathbf{0}$ extends the action of $\mathcal{G}_{\eta}$ to $B\left(\mathscr{H}_{3}\right)$ and $\|\bullet\|_{1 \rightarrow 1}$ is the induced Schatten $(1 \rightarrow 1)$ norm [61]. The verifier supplies to the prover a randomly chosen valid $\eta$, a positive integer $N$ sufficiently large for the process tomography [62] and copies of the quantum states $\sigma_{i}$ encoding $\rho_{i}$ on demand, where $\left\{\rho_{i}\right\}$ is chosen based on the tomography procedure in use. The prover returns $N$ copies of the qutrit states on which the change of inner product is successful as well as the success ratios for each $\rho_{i}$, both of which are used by the verifier to reconstruct $\hat{\mathcal{G}_{\eta}}$. To ensure that the verifier does not accept the process performed by a dishonest prover implementing only qubit-unitary channels and randomly discarding the system, it suffices to set the threshold to $D_{\text {th }}(\eta)=1 / 3\left(\lambda_{1}-\lambda_{2}\right)$, where $\lambda_{1}>\lambda_{2}>0$ are the eigenvalues of $\eta$ (see Appendix G).

In conclusion, we have three major results. First, we have operationalized Hilbert-space inner-product change in a way that is both observable and fully compatible with axiomatic quantum mechanics. Physically we can understand this innerproduct change as a lossy quantum operation effecting a change in norm. This lossy operation is reminiscent of how superluminality is reconciled by electromagnetic absorption [63], with loss in our case forbidding past counterfactual claims. Consistency of our work is proven using $\mathrm{C}^{*}$ algebra and representations. Alternatively, our claims can be verified experimentally by conducting two physically distinct experiments. One experiment is for the lower-dimensional lossy quantum operation and the other experiment is for the higher-dimensional unitary channel with both realizations 
yielding the same success ratio and measurement statistics for a given task. Our theory fully explains unbroken PTsymmetric quantum mechanics in all its forms as being about changing Hilbert-space inner product and observing its consequences. Our scheme for simulating qubit PT-symmetric Hamiltonians only requires one extra Hilbert-space dimension and no interaction with the environment, which eliminates the requirements for multiple subsystems and entangling operations used in existing schemes $[13,41,44,47,48]$. We also show how to simulate $d$-dimensional $(d \geqslant 2)$ PT-symmetric Hamiltonians using $2 d$ dimensions, as opposed to using $d^{3}$ dimensions in the Stinespring dilation approach. Our results open possibilities for simulating PT-symmetric dynamics on new experimental platforms, such as transmons, where high fidelity qutrit-unitary operations have already been demonstrated $[64,65]$.

This project is supported by the Government of Alberta and by the Natural Sciences and Engineering Research Council of Canada (NSERC). S.K. is grateful for a University of Calgary Eyes High International Doctoral Scholarship and an Alberta Innovates Graduate Student Scholarship. A.A. acknowledges support by Killam Trusts (Postdoctoral Fellowship).

\section{APPENDIX A: CONSTRUCTING A REPRESENTATION OF THE C* ALGEBRA ON THE HILBERT SPACE WITH A DIFFERENT INNER PRODUCT}

In this section, we show the construction of the Hilbert space $\mathscr{H}_{\eta}$ with inner product related to that of $\mathscr{H}$ by the metric operator $\eta$, the construction of a ${ }^{*}$-representation of the $\mathrm{C}^{*}$ algebra $\mathcal{A}$ on this new Hilbert space, and finally the representation of states in $\mathcal{S}$ using density operators on $\mathscr{H}_{\eta}$.

\section{Constructing a new Hilbert space from the metric operator}

For a possibly infinite dimensional Hilbert space $\mathscr{H}$, we denote by $\mathcal{L}(\mathscr{H})$ and $\mathcal{B}(\mathscr{H})$ the algebra of linear and bounded linear operators on $\mathscr{H}$ respectively. We also denote by $\mathcal{D}(\mathscr{H}):=\left\{\rho \in \mathcal{B}(\mathscr{H}): \rho \geqslant 0, \rho^{\dagger}=\rho, \operatorname{tr} \rho \leqslant 1\right\}$ the set of density operators acting on $\mathscr{H}_{\eta}$.

Definition 1 ([55]). The adjoint of an operator $A \in \mathcal{B}(\mathscr{H})$ is the unique operator $A^{\dagger} \in \mathcal{B}(\mathscr{H})$ satisfying

$$
\langle\phi|A| \psi\rangle=\overline{\left\langle\psi\left|A^{\dagger}\right| \phi\right\rangle} \forall|\phi\rangle,|\psi\rangle \in \mathscr{H} .
$$

The operator $A$ is self-adjoint if $A=A^{\dagger}$.

Definition 2 ([55]). An operator $A \in \mathcal{L}(\mathscr{H})$ is positivedefinite if

$$
\langle\phi|A| \phi\rangle>0 \quad \forall|\phi\rangle \in \mathscr{H},|\phi\rangle \neq 0 .
$$

The following theorem is adapted from the Appendix A of Ref. [26].

Theorem 1. For any Hilbert space $\mathscr{H}=(\mathscr{V},\langle\mid\rangle)$ and a self-adjoint, positive-definite operator $\eta \in \mathcal{B}(\mathscr{H})$,

1. the sesquilinear form

$$
\langle\bullet \mid \bullet\rangle_{\eta}:=\langle\bullet|\eta| \bullet\rangle
$$

is nondegenerate, therefore an inner product on $\mathscr{V}$.
2. The vector space $\mathscr{V}$ is complete with respect to the norm induced by the inner product $\langle\bullet \mid \bullet\rangle^{\prime}$, therefore $\mathscr{H}_{\eta}=$ $\left(\mathscr{V},\langle\mid\rangle_{\eta}\right)$ is a Hilbert space.

\section{Constructing a *-representation on the new Hilbert space}

We now construct a ${ }^{*}$-representation of the algebra $\mathcal{A}$ on the new Hilbert space $\mathscr{H}_{\eta}$ constructed in Theorem 1 . In the following, $\mathscr{H}$ and $\mathscr{H}_{\eta}$ are two Hilbert spaces with their inner product related by the metric operator $\eta$ as in Theorem 1 , $\mathcal{A}$ is a $\mathrm{C}^{*}$ algebra of operators and $\pi: \mathcal{A} \rightarrow \mathcal{B}(\mathscr{H})$ is a *representation of $\mathcal{A}$. We first establish some results required for constructing such a new representation. The following lemma, which establishes the inverse of the metric operator $\eta$, is adapted from the Appendix A of Ref. [26].

Lemma 2. Any self-adjoint and positive-definite operator $\eta \in \mathcal{B}(\mathscr{H})$ is invertible. Furthermore, the inverse $\eta^{-1} \in$ $\mathcal{B}(\mathscr{H})$ is self-adjoint and positive-definite.

We next show that the bounded operator spaces on $\mathscr{H}$ and $\mathscr{H}_{\eta}$ coincide.

Lemma 3. $M \in \mathcal{B}(\mathscr{H})$ if and only if $M \in \mathcal{B}\left(\mathscr{H}_{\eta}\right)$.

Proof. Let $\|\bullet\|,\|\bullet\|_{\eta}$ respectively denote the operator norms in $\mathscr{H}, \mathscr{H}_{\eta}$. From Eq. (A3),

$$
\|M\|_{\eta}=\left\|\eta^{1 / 2} M \eta^{-1 / 2}\right\|, \forall M \in \mathcal{B}(\mathscr{H}) .
$$

If $M \in \mathcal{B}(\mathscr{H})$, then

$$
\|M\|_{\eta} \leqslant\left\|\eta^{1 / 2}\right\| \cdot\|M\| \cdot\left\|\eta^{-1 / 2}\right\|<\infty
$$

and therefore, $M \in \mathcal{B}\left(\mathscr{H}_{\eta}\right)$. To verify the reverse implication, note that

$$
\begin{aligned}
\|M\| & =\left\|\eta^{-1 / 2}\left(\eta^{1 / 2} M \eta^{-1 / 2}\right) \eta^{1 / 2}\right\| \\
& \leqslant\left\|\eta^{-1 / 2}\right\| \cdot\|M\|_{\eta} \cdot\left\|\eta^{1 / 2}\right\|<\infty
\end{aligned}
$$

for all $M \in \mathcal{B}\left(\mathscr{H}_{\eta}\right)$.

The next lemma relates ${ }^{\dagger}$ to ${ }^{\ddagger}$, with the latter denoting the adjoint with respect to the inner product $\langle\bullet \mid \bullet\rangle^{\prime}$ of $\mathscr{H}_{\eta}$.

Lemma 4. For any $M \in \mathcal{B}(\mathscr{H}), M^{\ddagger}=\eta^{-1} M^{\dagger} \eta$. Additionally, $\eta^{\ddagger}=\eta$.

Proof. By definition of $\ddagger$, we have

$$
\begin{aligned}
\langle\phi|M| \psi\rangle_{\eta}=\overline{\left\langle\psi\left|M^{\ddagger}\right| \phi\right\rangle_{\eta}} & , \forall|\psi\rangle,|\phi\rangle \in \mathscr{V}, \\
& \forall M \in \mathcal{B}(\mathscr{H}) .
\end{aligned}
$$

Using Eq. (A3),

$$
\begin{gathered}
\langle\phi|M| \psi\rangle_{\eta}=\langle\phi|\eta M| \psi\rangle=\overline{\left\langle\psi\left|M^{\dagger} \eta\right| \phi\right\rangle}=\overline{\left\langle\psi\left|\eta^{-1} M^{\dagger} \eta\right| \phi\right\rangle_{\eta}}, \\
\forall|\psi\rangle,|\phi\rangle \in \mathscr{V}, \forall M \in \mathcal{B}(\mathscr{H}) .
\end{gathered}
$$

Then $M^{\ddagger}=\eta^{-1} M^{\dagger} \eta$ follows from the comparison of Eq. (A7) and Eq. (A8), and $\eta^{\ddagger}=\eta$ can be obtained by substituting $M=$ $\eta$ in this relation.

We are now ready for the construction of a *-representation of $\mathcal{A}$ on $\mathscr{H}_{\eta}$.

Theorem 5. Let $\pi: \mathcal{A} \rightarrow \mathcal{B}(\mathscr{H})$ be a ${ }^{*}$-representation of a $\mathrm{C}^{*}$ algebra $\mathcal{A}$. Then $\pi_{\eta}: \mathcal{A} \rightarrow \mathcal{L}\left(\mathscr{H}_{\eta}\right): A \mapsto \eta^{-1 / 2} \pi(A) \eta^{1 / 2}$ is a ${ }^{*}$-representation of $\mathcal{A}$ on $\mathscr{H}_{\eta}$.

Proof. The range of the map $\pi_{\eta}$ is $\mathcal{B}\left(\mathscr{H}_{\eta}\right)$, which follows from the fact that $\eta^{1 / 2}, \eta^{-1 / 2}, \pi(A) \in \mathcal{B}\left(\mathscr{H}_{\eta}\right)$, following Lemma 3 and definition of $\pi$, for all $A \in$ $\mathcal{A}$. The map $\pi_{\eta}$ is linear by construction, and it 
is product preserving because $\pi_{\eta}(A B)=\eta^{-1 / 2} \pi(A B) \eta^{1 / 2}=$ $\eta^{-1 / 2} \pi(A)\left(\eta^{1 / 2} \eta^{-1 / 2}\right) \pi(B) \eta^{1 / 2}=\pi_{\eta}(A) \pi_{\eta}(B)$. Therefore $\pi_{\eta}$ is a representation. The representation $\pi_{\eta}$ is also *-preserving, as

$$
\begin{aligned}
\pi_{\eta}\left(A^{*}\right) & =\eta^{-1 / 2} \pi\left(A^{*}\right) \eta^{1 / 2}=\eta^{-1 / 2} \pi(A)^{\dagger} \eta^{1 / 2}=\eta^{1 / 2} \pi(A)^{\ddagger} \eta^{-1 / 2} \\
& =\left(\eta^{-1 / 2} \pi(A) \eta^{1 / 2}\right)^{\ddagger}=\pi_{\eta}(A)^{\ddagger} .
\end{aligned}
$$

Therefore $\pi_{\eta}$ is a ${ }^{*}$-representation of $\mathcal{A}$ on $\mathscr{H}_{\eta}$.

\section{Representing states on the new Hilbert space}

We now characterize the set of states represented by the set of density operators $\mathcal{D}\left(\mathscr{H}_{\eta}\right)$ and construct vector representation of the pure states under $\pi_{\eta}$. Recall that ${ }^{\#} \pi: \rho \mapsto \omega$ such that $\omega(A)=\operatorname{tr}(\rho \pi(A)) \forall A \in \mathcal{A}$. The map ${ }^{\#} \pi_{\eta}$ is defined analogously for the $\pi_{\eta}$ representation. In the following lemma we show how the density operators acting on $\mathscr{H}$ and $\mathscr{H}_{\eta}$ are related, which we further use to prove that the set of states represented under $\pi_{\eta}$ coincides with that represented under $\pi$, namely $\mathcal{S}$.

Lemma 6. An operator $\rho_{\eta} \in \mathcal{D}\left(\mathscr{H}_{\eta}\right)$ if and only if

$$
\rho_{\eta}=\eta^{-1 / 2} \rho \eta^{1 / 2}
$$

for some $\rho \in \mathcal{D}(\mathscr{H})$.

Proof. Let $B:=\left\{\left|e_{i}\right\rangle\right\}$ be an orthonormal basis of $\mathscr{H}$. We first show that $B_{\eta}:=\left\{\left|f_{i}\right\rangle:=\eta^{-1 / 2}\left|e_{i}\right\rangle\right\}$ is an orthonormal basis of $\mathscr{H}_{\eta}$. The orthonormality of $\left\{\left|f_{i}\right\rangle\right\}$ follows from $\left\langle f_{i} \mid f_{j}\right\rangle^{\prime}=\left\langle e_{i}\left|\eta^{-1 / 2} \eta \eta^{-1 / 2}\right| e_{j}\right\rangle^{\prime}=\delta_{i j}$. We prove that $\left\{\left|f_{i}\right\rangle\right\}$ is a basis by showing that any $|\phi\rangle \in \mathscr{V}$ can be expressed as $|\phi\rangle=\sum_{i}\left\langle f_{i} \mid \phi\right\rangle^{\prime}\left|f_{i}\right\rangle$. Let $|\psi\rangle=\eta^{1 / 2}|\phi\rangle$. Then $|\psi\rangle=$ $\sum_{i}\left\langle e_{i} \mid \psi\right\rangle\left|e_{i}\right\rangle$. Now premultiplying by $\eta^{-1 / 2}$ and substituting $\left|f_{i}\right\rangle=\eta^{-1 / 2}\left|e_{i}\right\rangle,|\psi\rangle=\eta^{1 / 2}|\phi\rangle$ yields the desired expression $|\phi\rangle=\sum_{i}\left\langle f_{i} \mid \phi\right\rangle^{\prime}\left|f_{i}\right\rangle$. Note that this sum is convergent because the set $\left\{\left|f_{i}\right\rangle\right\}$ is orthonormal [55].

To prove the forward implication, we note that Hilbert Schmidt norm of any $\rho \in \mathcal{D}(\mathscr{H})$ is finite, i.e., $\sum_{i \in B} \| \rho\left|e_{i}\right\rangle \|^{2}<\infty$, and $\operatorname{tr}(\rho)=\sum_{i \in B}\left\langle e_{i}|\rho| e_{i}\right\rangle<\infty$; both these properties follow from $\rho$ being a trace-class operator [55]. For $\rho_{\eta} \in \mathcal{B}\left(\mathscr{H}_{\eta}\right)$ satisfying Eq. (A10),

$$
\begin{aligned}
\sum_{i \in B_{\eta}} \| \rho_{\eta}\left|f_{i}\right\rangle \|_{\eta}^{2} & =\sum_{i \in B} \| \eta^{-1 / 2} \rho\left|e_{i}\right\rangle \|_{\eta}^{2} \\
& =\sum_{i \in B}\left\langle e_{i}\left|\left(\eta^{-1 / 2} \rho\right)^{\dagger} \eta\left(\eta^{-1 / 2} \rho\right)\right| e_{i}\right\rangle \\
& =\sum_{i \in B} \| \rho\left|e_{i}\right\rangle \|^{2}<\infty
\end{aligned}
$$

Therefore, $\rho_{\eta}$ is a Hilbert-Schmidt operator in $\mathcal{B}\left(\mathscr{H}_{\eta}\right)$. Furthermore,

$$
\begin{aligned}
\operatorname{tr}\left(\rho_{\eta}\right) & =\sum_{i \in B_{\eta}}\left\langle f_{i}\left|\rho_{\eta}\right| f_{i}\right\rangle_{\eta}=\sum_{i \in B}\left\langle e_{i}\left|\eta^{-1 / 2} \eta \rho_{\eta} \eta^{-1 / 2}\right| e_{i}\right\rangle \\
& =\sum_{i \in B}\left\langle e_{i}|\rho| e_{i}\right\rangle=\operatorname{tr}(\rho) .
\end{aligned}
$$

Therefore, $\rho_{\eta}$ is a trace-class operator with $\operatorname{tr}\left(\rho_{\eta}\right) \leqslant 1$ and hence $\rho_{\eta} \in \mathcal{D}\left(\mathscr{H}_{\eta}\right)$. Similarly, the reverse implication that for any $\rho_{\eta} \in \mathcal{D}\left(\mathscr{H}_{\eta}\right)$, the operator $\eta^{1 / 2} \rho_{\eta} \eta^{-1 / 2} \in \mathcal{D}(\mathscr{H})$ is proved by starting with an orthonormal basis $\left\{\left|f_{i}\right\rangle\right\}$ for $\mathscr{H}_{\eta}$ and observing that $\left\{\eta^{1 / 2}\left|f_{i}\right\rangle\right\}$ is an orthornormal basis for $\mathscr{H}$.
We now show that both $\mathcal{D}(\mathscr{H})$ and $\mathcal{D}\left(\mathscr{H}_{\eta}\right)$ represent the same set of states, $\mathcal{S}$, under the respective *-representations.

Lemma 7. $\rho_{\eta} \stackrel{\#_{\pi_{\eta}}}{\rightarrow} \omega$ if and only if $\rho \stackrel{\#_{\pi}}{\rightarrow} \omega$, where $\rho, \rho_{\eta}$ are related by Eq. (A10).

Proof. To prove the forward implication, note $\omega(A)=$ $\operatorname{tr}(\rho \pi(A)) \forall A$ by the definition of ${ }^{\#} \pi$. Then $\operatorname{tr}(\rho \pi(A))=$ $\operatorname{tr}\left(\rho_{\eta} \eta^{-1 / 2} \pi(A) \eta^{1 / 2}\right)=\operatorname{tr}\left(\rho_{\eta} \pi_{\eta}(A)\right)$ using the cyclic property of trace and the definition of $\pi_{\eta}$ respectively. Therefore $\omega(A)=$ $\operatorname{tr}\left(\rho_{\eta} \pi_{\eta}(A)\right) \forall A$, and therefore, $\rho_{\eta} \stackrel{{ }^{{ }^{*} \pi_{\eta}} \rightarrow}{\rightarrow} \omega$. The reverse implication can be proved by following the same steps in reverse order.

Finally, we represent pure states in $\mathcal{S}$ by vectors in the Hilbert space $\mathscr{H}_{\eta}$. Recall that a state $\omega \in \mathcal{S}$ has a vectorrepresentation $|\psi\rangle \in \mathscr{H}$ under $\pi$ if

$$
\omega(A)=\langle\psi|\pi(A)| \psi\rangle \quad \forall A \in \mathcal{A} .
$$

We now extend this definition to the representation $\pi_{\eta}$.

Definition 3. A state $\omega \in \mathcal{S}$ has a vector representation $|\psi\rangle \in \mathscr{H}_{\eta}$ under $\pi_{\eta}$ if

$$
\omega(A)=\left\langle\psi\left|\pi_{\eta}(A)\right| \psi\right\rangle_{\eta} \quad \forall A \in \mathcal{A} .
$$

Let the ball $\overline{B_{1}}(\mathscr{H}):=\{|\psi\rangle \in \mathscr{H}: \sqrt{\langle\psi \mid \psi\rangle} \leqslant 1\}$ denote the set of normalized and subnormalized vectors in $\mathscr{H}$. Recall that the map lift $: \overline{B_{1}}(\mathscr{H}) \rightarrow \mathcal{D}(\mathscr{H}):|\psi\rangle \mapsto|\psi\rangle\langle\psi|$ connects the vector representation $|\psi\rangle$ of a pure state $\omega$ to its density operator representation $|\psi\rangle\langle\psi|$ under $\pi$. We now construct an analogous map lift ${ }_{\eta}: \overline{B_{1}}\left(\mathscr{H}_{\eta}\right) \rightarrow \mathcal{D}\left(\mathscr{H}_{\eta}\right)$ for the representation $\pi_{\eta}$, where the ball $\overline{B_{1}}\left(\mathscr{H}_{\eta}\right):=\left\{|\psi\rangle \in \mathscr{H}_{\eta}\right.$ : $\left.\sqrt{\langle\psi \mid \psi\rangle}_{\eta} \leqslant 1\right\}$.

Definition 4. The map lift $\operatorname{li}_{\eta}: \overline{B_{1}}\left(\mathscr{H}_{\eta}\right) \rightarrow \mathcal{D}\left(\mathscr{H}_{\eta}\right)$ is defined to be the map that satisfies the following condition: for any state $\omega \in \mathcal{S}$ with vector representation $|\psi\rangle \in \mathscr{H}_{\eta}$ and density operator representation $\rho_{\eta} \in \mathcal{D}\left(\mathscr{H}_{\eta}\right)$, lift ${ }_{\eta}:|\psi\rangle \mapsto \rho_{\eta}$.

We now derive the explicit action of lift $\eta_{\eta}$.

Lemma 8. The map lift ${ }_{\eta}$ has action $\operatorname{lift}_{\eta}: \overline{B_{1}}\left(\mathscr{H}_{\eta}\right) \rightarrow$ $\mathcal{D}\left(\mathscr{H}_{\eta}\right):|\psi\rangle \mapsto|\psi\rangle\langle\psi| \eta$.

Proof. Let lift ${ }_{\eta}:|\psi\rangle \mapsto \rho_{\eta}$ and ${ }^{\#} \pi_{\eta}: \rho_{\eta} \mapsto \omega$. Following the definition of ${ }^{\#} \pi_{\eta}$ and lift $_{\eta}$,

$$
\left\langle\psi\left|\pi_{\eta}(A)\right| \psi\right\rangle_{\eta}=\operatorname{tr}\left(\rho_{\eta} \pi_{\eta}(A)\right)=\omega(A) \quad \forall A .
$$

As $\quad\left\langle\psi\left|\pi_{\eta}(I)\right| \psi\right\rangle_{\eta}=\omega(I) \leqslant 1, \quad|\psi\rangle \in \overline{B_{1}}\left(\mathscr{H}_{\eta}\right)$. Using Eq. (A3), we get $\left\langle\psi\left|\pi_{\eta}(A)\right| \psi\right\rangle_{\eta}=\left\langle\psi\left|\eta \pi_{\eta}(A)\right| \psi\right\rangle$. Then using the cyclic property of the trace, this expectation value can be expressed as

$$
\left\langle\psi\left|\eta \pi_{\eta}(A)\right| \psi\right\rangle=\operatorname{tr}\left(|\psi\rangle\langle\psi| \eta \pi_{\eta}(A)\right) \quad \forall A,
$$

therefore, $\rho_{\eta}=|\psi\rangle\langle\psi| \eta \in \mathcal{D}\left(\mathscr{H}_{\eta}\right)$. This leads to the desired action of lift $\eta$.

\section{APPENDIX B: QUANTUM OPERATION FOR IMPLEMENTING THE CHANGING INNER PRODUCT}

In this section, we construct the quantum operation that implements the change in inner product by $\eta \leqslant \pi(I)$. Change in inner product is defined by the identity isomorphism $\mathcal{I}_{\eta}$ : $\mathscr{H} \rightarrow \mathscr{H}_{\eta}$ [see Fig. 1(c) in main text]. We now show how $\mathcal{I}_{\eta}$ is extended to $\mathcal{B}\left(\mathscr{H}_{\eta}\right)$ through the map $\mathcal{E}_{\eta}$ defined below. 
Theorem 9. Let $\eta \leqslant \pi(I)$ and $\mathcal{E}_{\eta}: \mathcal{B}(\mathscr{H}) \rightarrow \mathcal{L}\left(\mathscr{H}_{\eta}\right)$ : $M \mapsto M \eta$. Then

1. $\operatorname{range}\left(\mathcal{E}_{\eta}\right) \subseteq \mathcal{B}\left(\mathscr{H}_{\eta}\right)$

2. $\mathcal{E}_{\eta}$ satisfies the following commutative diagram:

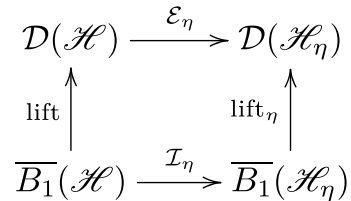

3. $\mathcal{E}_{\eta}$ is a quantum operation.

Proof. To prove Statement 1, note that for any $M \in \mathcal{B}(\mathscr{H})$, the operator $M \eta \in \mathcal{B}\left(\mathscr{H}_{\eta}\right)$ because

$$
\|M \eta\|_{\eta}=\left\|\eta^{1 / 2}(M \eta) \eta^{-1 / 2}\right\| \leqslant\left\|\eta^{1 / 2}\right\|^{2} \cdot\|M\| \leqslant \infty,
$$

where the first equality follows from Eq. (A4). The commutative diagram in Statement 2 follows immediately from the action of lift $\eta_{\eta}$ map in Lemma 8.

We now show that $\mathcal{E}_{\eta}$ is a valid quantum operation, i.e., a completely-positive, trace nonincreasing map. To prove the positivity of $\mathcal{E}_{\eta}$, let $M \geqslant 0$, so that it can be expressed as $M=A A^{\dagger}$ [55]. Then $\mathcal{E}_{\eta}(M)=A A^{\dagger} \eta$, which can expressed as $\mathcal{E}_{\eta}(M)=B B^{\ddagger}$ with $B=A \eta^{\frac{1}{2}}$. Therefore $\mathcal{E}_{\eta}(M) \in \mathcal{B}\left(\mathscr{H}_{\eta}\right)$ is positive if $M$ is positive, which proves the positivity of $\mathcal{E}_{\eta}$.

Complete positivity of $\mathcal{E}_{\eta}$ can be proven by showing that the map $\mathcal{E}_{\eta} \otimes \mathscr{I}_{k}: \mathcal{B}(\mathscr{H}) \otimes \mathcal{B}\left(\mathbb{C}^{k}\right) \rightarrow \mathcal{B}\left(\mathscr{H}_{\eta}\right) \otimes \mathcal{B}\left(\mathbb{C}^{k}\right)$ is positive, for every positive integer $k$, where $\mathscr{I}_{k}$ denotes the identity map on $\mathcal{B}\left(\mathbb{C}^{k}\right)$. The action of the new map is given by $\left[\mathcal{E}_{\eta} \otimes \mathscr{I}_{k}\right](N)=N\left(\eta \otimes I_{k}\right)$, with $I_{k} \in \mathcal{B}\left(\mathbb{C}^{k}\right)$ the identity operator. Operator $\left[\mathcal{E}_{\eta} \otimes \mathscr{I}_{k}\right](N) \in \mathcal{B}\left(\mathscr{H}_{\eta}\right) \otimes \mathcal{B}\left(\mathbb{C}^{k}\right)$ because $\left\|\eta \otimes I_{k}\right\|=\|\eta\|_{\eta} \cdot\left\|I_{k}\right\|=\|\eta\|_{\eta}$. For proving positivity, let $N \in \mathcal{B}(\mathscr{H}) \otimes \mathcal{B}\left(\mathbb{C}^{k}\right)$ be a positive operator, so that $N=C C^{\dagger}$. Then $\left[\mathcal{E}_{\eta} \otimes \mathscr{I}_{k}\right](N)=C C^{\dagger}\left(\eta \otimes I_{k}\right)$, which can be expressed as $\left[\mathcal{E}_{\eta} \otimes \mathscr{I}_{k}\right](N)=D D^{\ddagger}$ with $D=C\left(\eta^{\frac{1}{2}} \otimes I_{k}\right)$, thereby proving positivity of $\left[\mathcal{E}_{\eta} \otimes \mathscr{I}_{k}\right](N)$ and consequently positivity of $\mathcal{E}_{\eta} \otimes \mathscr{I}_{k}$.

To prove that $\mathcal{E}_{\eta}$ is trace nonincreasing, let $\rho \in \mathcal{D}(\mathscr{H})$ and note that trace is independent of the inner product [Eq. (A12)]. We have $\operatorname{tr}(\rho \eta)=\operatorname{tr}\left(\eta^{1 / 2} \rho \eta^{1 / 2}\right)$ and

$$
\operatorname{tr}\left(\eta^{1 / 2} \rho \eta^{1 / 2}\right)=\operatorname{tr}\left|\eta^{1 / 2} \rho \eta^{1 / 2}\right| \leqslant\left\|\eta^{1 / 2}\right\|^{2} \operatorname{tr}|\rho| \leqslant \operatorname{tr}(\rho),
$$

where $|M|=\sqrt{\left(M^{\dagger} M\right)}$ and we used $|M|=M$ for any $M \geqslant 0$. The first inequality in Eq. (B3) is a property of the trace norm [55], and the last inequality in Eq. (B3) follows from the fact that $\eta \leqslant \pi(I)$ and therefore, $\left\|\eta^{1 / 2}\right\|^{2} \leqslant 1$.

\section{APPENDIX C: TRANSFORMATION OF THE OPERATORS UNDER CHANGING THE INNER PRODUCT}

An inner product changing channel could modify the commutation relations between the operators. In this section, we demonstrate such a change with an explicit example of a qubit system undergoing an inner-product change. Consider a qubit system undergoing change in inner product by

$$
\eta=\frac{1}{1+r \sin \phi}\left(\begin{array}{cc}
1 & -\mathrm{i} r \sin \phi \\
\mathrm{i} r \sin \phi & 1
\end{array}\right), \quad 0 \leqslant r<1 .
$$

The Pauli operators $X, Y, Z \in \mathcal{B}(\mathscr{H})$ acting on the original Hilbert space along with the identity operator $I_{2} \in \mathcal{B}(\mathscr{H})$ generate the $\mathfrak{u}(2)$ algebra. These operators transform according to Eq. (5) in the main text following the inner-product change by $\eta$. This transformation is given by the map $\mathcal{E}_{\eta}^{\mathrm{op}}$. The transformed operators satisfy the commutation relations

$$
\begin{aligned}
& {\left[\mathcal{E}_{\eta}^{\mathrm{op}}(X), \mathcal{E}_{\eta}^{\mathrm{op}}(Y)\right]=2 \mathrm{i} a \mathcal{E}_{\eta}^{\mathrm{op}}(Z),} \\
& {\left[\mathcal{E}_{\eta}^{\mathrm{op}}\left(I_{2}\right), \mathcal{E}_{\eta}^{\mathrm{op}}(Z)\right]=2 \mathrm{i}(1-a) \mathcal{E}_{\eta}^{\mathrm{op}}(X),} \\
& {\left[\mathcal{E}_{\eta}^{\mathrm{op}}(Y), \mathcal{E}_{\eta}^{\mathrm{op}}(Z)\right]=2 \mathrm{i} a \mathcal{E}_{\eta}^{\mathrm{op}}(X),} \\
& {\left[\mathcal{E}_{\eta}^{\mathrm{op}}\left(I_{2}\right), \mathcal{E}_{\eta}^{\mathrm{op}}(X)\right]=-2 \mathrm{i}(1-a) \mathcal{E}_{\eta}^{\mathrm{op}}(Z),} \\
& {\left[\mathcal{E}_{\eta}^{\mathrm{op}}(Z), \mathcal{E}_{\eta}^{\mathrm{op}}(X)\right]=-2 \mathrm{i}(1-a) \mathcal{E}_{\eta}^{\mathrm{op}}\left(I_{2}\right)+2 \mathrm{i} a \mathcal{E}_{\eta}^{\mathrm{op}}(Y),} \\
& {\left[\mathcal{E}_{\eta}^{\mathrm{op}}\left(I_{2}\right), \mathcal{E}_{\eta}^{\mathrm{op}}(Y)\right]=\mathbf{0},}
\end{aligned}
$$

where $a=1 /(1+r \sin \phi)$. These commutation relations are different from those of $\mathfrak{u}(2)$ algebra for $r \neq 0$, or equivalently $a \neq 1$.

\section{APPENDIX D: MATRIX REPRESENTATION OF THE QUTRIT UNITARY OPERATOR THAT SIMULATES CHANGE IN INNER PRODUCT OF A QUBIT SYSTEM}

In this section, we derive the matrix representation of the qutrit unitary operator $U_{\tilde{\eta}}$ [see Eq. (12) in main text] employed in the simulation of the change in inner product of a qubit system. Equation (10) in the main text requires that $P U_{\tilde{\eta}} P=$ $\tilde{\eta}^{1 / 2}$, so that $U_{\tilde{\eta}}$ can be expressed as

$$
\left[U_{\tilde{\eta}}\right]=\left(\begin{array}{cc}
{[\tilde{\eta}]^{\frac{1}{2}}} & \boldsymbol{u} \\
\overline{\boldsymbol{v}}^{\top} & \boldsymbol{r} \mathrm{e}^{\mathrm{i} \theta}
\end{array}\right)
$$

for some vectors $\boldsymbol{u}, \boldsymbol{v} \in \mathbb{C}^{2}$, a number $r \in[0,1]$ and a phase $\theta \in[0,2 \pi)$. The unitarity conditions $U_{\tilde{\eta}}^{\dagger} U_{\tilde{\eta}}=U_{\tilde{\eta}} U_{\tilde{\eta}}^{\dagger}=I_{3}$ lead to

$$
\begin{gathered}
{[\tilde{\eta}]+\boldsymbol{u} \overline{\boldsymbol{u}}^{\top}=I_{2},} \\
{[\tilde{\eta}]^{\frac{1}{2}} \boldsymbol{u}+r \mathrm{e}^{\mathrm{i} \theta} \boldsymbol{v}=0,} \\
\overline{\boldsymbol{u}}^{\top} \boldsymbol{u}+r^{2}=1 \Rightarrow\|\boldsymbol{u}\|=1-r^{2} .
\end{gathered}
$$

Postmultiplying Eq. (D2) by $\boldsymbol{u}$ and substituting Eq. (D4) yields $[\tilde{\eta}] \boldsymbol{u}=r^{2} \boldsymbol{u}$, which implies that $\boldsymbol{u}$ is the eigenvector of $[\tilde{\eta}]^{1 / 2}$ with eigenvalue $r$. Then Eq. (D3) yields $\boldsymbol{v}=-\mathrm{e}^{-\mathrm{i} \theta} \boldsymbol{u}$ as desired, with the global phase of $\boldsymbol{u}$ and $\theta$ being the free parameters.

\section{APPENDIX E: SIMULATION OF A QUBIT PT-SYMMETRIC HAMILTONIAN USING SINGLE QUTRIT}

We now design a qutrit procedure that simulates the dynamics of a qubit Hamiltonian with unbroken PT symmetry. Our design is based on the qutrit procedure for simulating the change in inner product of a qubit system, provided in the main text (Fig. 4). We illustrate our Hamiltonian-simulation procedure using the PT-symmetric Hamiltonian $H_{\mathrm{PT}}$ from [3].

The matrix form of $H_{\mathrm{PT}}$ is

$$
\left[H_{\mathrm{PT}}\right]=\left(\begin{array}{cc}
r \mathrm{e}^{\mathrm{i} \phi} & s \\
s & r \mathrm{e}^{-\mathrm{i} \phi}
\end{array}\right), \quad s>r \sin \phi \geqslant 0 .
$$


Dynamics generated by $H_{\mathrm{PT}}$ is denoted by the operator $\mathcal{U}_{\mathrm{PT}}$ [Eq. (6) in main text],

$$
\rho \stackrel{\mathcal{U}_{\mathrm{PT}}}{\mapsto} \kappa U_{\mathrm{PT}} \rho U_{\mathrm{PT}}^{\dagger}, U_{\mathrm{PT}}:=\mathrm{e}^{-\mathrm{i} H_{\mathrm{PT}} t / \hbar}, \kappa=\frac{1}{\left\|\eta_{2}^{-1}\right\|} .
$$

As proved in the main text, $\mathcal{U}_{\mathrm{PT}}$ can be expressed as a sequence of operations acting exclusively on $\mathcal{B}\left(\mathscr{H}_{2}\right)$,

$$
\mathcal{U}_{\mathrm{PT}}=\mathcal{G}_{\kappa \eta_{2}^{-1}} \circ\left(\mathcal{R}_{\kappa \eta_{2}^{-1}} \circ \tilde{\mathcal{U}}_{\mathrm{PT}} \circ \mathcal{R}_{\eta_{2}}\right) \circ \mathcal{G}_{\eta_{2}},
$$

where $\mathcal{R}_{\kappa \eta^{-1}} \circ \tilde{\mathcal{U}}_{\mathrm{PT}} \circ \mathcal{R}_{\eta}: \mathcal{B}\left(\mathscr{H}_{2}\right) \rightarrow \mathcal{B}\left(\mathscr{H}_{2}\right)$ is the channel with unitary Kraus operator $\eta^{1 / 2} U_{\mathrm{PT}} \eta^{-1 / 2}$ and the Kraus operator is generated by self-adjoint Hamiltonian $h_{\mathrm{PT}}=$ $\eta^{1 / 2} H_{\mathrm{PT}} \eta^{-1 / 2}$.

Our qutrit procedure for simulating $\mathcal{U}_{\mathrm{PT}}$ involves implementing each operation in Eq. (E3) using qutrit unitaries and measurements, as we now explain through Steps 1-4. The input to the simulation procedure is $\rho \in \mathcal{B}\left(\mathscr{H}_{2}\right)$ embedded as $\sigma:=\rho \oplus \mathbf{0} \in \mathcal{B}\left(\mathscr{H}_{3}\right)$ and a time $t>0$. The output of the procedure is the state $\frac{U_{\mathrm{PT} \rho} U_{\mathrm{PT}}^{\dagger}}{\operatorname{tr}\left(U_{\mathrm{PT}} \rho U_{\mathrm{PT}}^{\dagger}\right)}$ with probability $\frac{1}{\left\|\eta_{2}^{-1}\right\|} \operatorname{tr}\left(U_{\mathrm{PT}} \rho U_{\mathrm{PT}}^{\dagger}\right)$. The simulation steps are

1. Calculate the metric operator and its inverse: The agent calculates $\eta_{2}, \eta_{2}^{-1}$ satisfying the quasi-Hermiticity condition $H_{\mathrm{PT}}^{\dagger}=\eta_{2} H_{\mathrm{PT}} \eta_{2}^{-1}$. A choice of $\eta_{2}$ and, therefore $\eta_{2}^{-1}$, is

$$
\begin{gathered}
{\left[\eta_{2}\right]=\frac{1}{s+r \sin \phi}\left(\begin{array}{cc}
s & -\mathrm{i} r \sin \phi \\
\mathrm{i} \sin \phi & s
\end{array}\right),} \\
{\left[\eta_{2}^{-1}\right]=\frac{1}{s-r \sin \phi}\left(\begin{array}{cc}
s & \mathrm{i} r \sin \phi \\
-\mathrm{i} r \sin \phi & s
\end{array}\right),}
\end{gathered}
$$

with $\left\|\eta_{2}\right\|=1$ and $\left\|\eta_{2}^{-1}\right\|=(s+r \sin \phi) /(s-r \sin \phi)$.

2. Simulate change in inner product by $\eta_{2}$ : Agent implements the qutrit procedure (Fig. 4) to simulate $\mathcal{G}_{\eta_{2}}$ by setting $\tilde{\eta}=\eta_{2}$ and for a single copy of $\sigma$. A choice of the qutrit unitary $U_{\eta_{2}}$ [see Eq. (D1)] simulating the action of $\mathcal{G}_{\eta_{2}}$ is

$$
U_{\eta_{2}}=\left(\begin{array}{ccc}
(1+q) / 2 & -\mathrm{i}(1-q) / 2 & p \\
\mathrm{i}(1-q) / 2 & (1+q) / 2 & -\mathrm{i} p \\
-p & -\mathrm{i} p & q
\end{array}\right),
$$

where

$$
q=\sqrt{\frac{s-r \sin \phi}{s+r \sin \phi}}=\frac{1}{\sqrt{\left\|\eta_{2}^{-1}\right\|}}, p=\sqrt{\frac{r \sin \phi}{s+r \sin \phi}} .
$$

The output of this step is the qutrit state $\frac{\eta_{2}^{1 / 2} \rho \eta_{2}^{1 / 2}}{\operatorname{tr}\left(\eta_{2}^{1 / 2} \rho \eta_{2}^{1 / 2}\right)} \oplus \mathbf{0}$ with probability $\operatorname{tr}\left(\eta_{2}^{1 / 2} \rho \eta_{2}^{1 / 2}\right)$.

3. Simulate the unitary evolution generated by $h_{\mathrm{PT}}$ : Agent calculates $h_{\text {PT }}$ embedded in $\mathcal{B}\left(\mathscr{H}_{3}\right)$,

$$
\left[h_{\mathrm{PT}} \oplus \mathbf{0}\right]=\left(\begin{array}{ccc}
r \cos \phi & \sqrt{s^{2}-r^{2} \sin ^{2} \phi} & 0 \\
\sqrt{s^{2}-r^{2} \sin ^{2} \phi} & r \cos \phi & 0 \\
0 & 0 & 0
\end{array}\right),
$$

and implements the qutrit unitary operator $e^{-\mathrm{i}\left(h_{\mathrm{PT}} \oplus \mathbf{0}\right) t}$, which is equivalent to simulating the channel $\left(\mathcal{R}_{\kappa \eta_{2}^{-1}} \circ \tilde{\mathcal{U}}_{\mathrm{PT}} \circ \mathcal{R}_{\eta_{2}}\right)$ in Eq. (E3). The output of this deterministic step is the qutrit state $\left(e^{-\mathrm{i} h_{\mathrm{PT}} t} \frac{\left(\eta_{2}^{1 / 2} \rho \eta_{2}^{1 / 2}\right)}{\operatorname{tr}\left(\eta_{2}^{1 / 2} \rho \eta_{2}^{1 / 2}\right)} e^{\mathrm{i} h_{\mathrm{PT}} t}\right) \oplus \mathbf{0}$, provided Step 2 is successful.
4. Simulate change in inner product by $\kappa \eta_{2}^{-1}$ : Agent applies the qutrit procedure (Fig. 4) to simulate $\mathcal{G}_{\kappa \eta_{2}^{-1}}$, by setting $\tilde{\eta}=\kappa \eta_{2}^{-1}$. Note that we have $\kappa=\frac{1}{\left\|\eta_{2}^{-1}\right\|}=q^{2}$ [Eqs. (E3) and (E6)]. A choice of $U_{\kappa \eta_{2}^{-1}}$ is

$$
U_{\kappa \eta_{2}^{-1}}=\left(\begin{array}{ccc}
(1+q) / 2 & \mathrm{i}(1-q) / 2 & p \\
-\mathrm{i}(1-q) / 2 & (1+q) / 2 & \mathrm{i} p \\
-p & \mathrm{i} p & q
\end{array}\right) .
$$

The output of this procedure is the qutrit state

$$
\frac{\left(\eta_{2}^{-1 / 2} e^{-\mathrm{i} h_{\mathrm{PT}} t} \eta_{2}^{1 / 2} \rho \eta_{2}^{1 / 2} e^{\mathrm{i} h_{\mathrm{PT}} t} \eta_{2}^{-1 / 2}\right)}{\operatorname{tr}\left(\eta_{2}^{-1 / 2} e^{-\mathrm{i} h_{\mathrm{PT}} t} \eta_{2}^{1 / 2} \rho \eta_{2}^{1 / 2} e^{\mathrm{i} h_{\mathrm{PT} t}} \eta_{2}^{-1 / 2}\right)} \oplus \mathbf{0}=\frac{U_{\mathrm{PT}} \rho U_{\mathrm{PT}}^{\dagger}}{\operatorname{tr}\left(U_{\mathrm{PT}} \rho U_{\mathrm{PT}}^{\dagger}\right)} \oplus \mathbf{0}
$$

with probability $\frac{\operatorname{tr}\left(U_{\mathrm{PT}} \rho U_{\mathrm{PT}}^{\dagger}\right)}{\left\|\eta_{2}^{-1}\right\| \operatorname{tr}\left(\eta_{2}^{1 / 2} \rho \eta_{2}^{1 / 2}\right)}$.

Therefore, the output of the simulation procedure is the state $\frac{U_{\mathrm{PT}} \rho U_{\mathrm{PT}}^{\dagger}}{\operatorname{tr}\left(U_{\mathrm{PT}} \rho U_{\mathrm{PT}}^{\dagger}\right)} \oplus \mathbf{0}$ with success probability given by the combined probability of success in Steps 2,4, which is equal to $\frac{1}{\left\|\eta_{2}^{-1}\right\|} \operatorname{tr}\left(U_{\mathrm{PT}} \rho U_{\mathrm{PT}}^{\dagger}\right)$.

\section{APPENDIX F: SIMULATION OF CHANGE IN INNER PRODUCT AND PT-SYMMETRIC DYNAMICS OF A $d$-DIMENSIONAL SYSTEM}

We first explain a simulation procedure to change the inner product of a $d$-dimensional system using $2 d$ dimensions. We assume that the algebra $\mathcal{A}$ of the system is represented on a $d$-dimensional Hilbert space $\mathscr{H}_{d}^{(s)}$ by $\pi$. Similar to the qutrit simulation procedure explained in main text, the agent simulating $\mathcal{G}_{\eta}$ for $\eta \leqslant \pi(I)$ first constructs the metric operator $\tilde{\eta}=\frac{1}{\|\eta\|} \eta$ and the unitary operator $U_{\tilde{\eta}} \in \mathcal{B}\left(\mathscr{H}_{d}^{(s)} \oplus \mathscr{H}_{d}^{(a)}\right)$ satisfying

$$
\mathcal{G}_{\tilde{\eta}}(\rho) \oplus \mathbf{0}=P U_{\tilde{\eta}} \sigma U_{\tilde{\eta}}^{\dagger} P, \sigma:=\rho \oplus \mathbf{0}, \forall \rho \in \mathcal{B}\left(\mathscr{H}_{d}^{(s)}\right),
$$

where $\mathbf{0}$ denotes the zero operator in $\mathcal{B}\left(\mathscr{H}_{d}^{(a)}\right)$. The matrix representation of a choice of $U_{\tilde{\eta}}$ is

$$
\left[U_{\tilde{\eta}}\right]=\left(\begin{array}{cc}
{[\tilde{\eta}]^{\frac{1}{2}}} & {[1-\tilde{\eta}]^{\frac{1}{2}}} \\
{[1-\tilde{\eta}]^{\frac{1}{2}}} & -[\tilde{\eta}]^{\frac{1}{2}}
\end{array}\right) .
$$

Agent then implements $U_{\tilde{\eta}}$ followed by projective measurement and postselection on to the subspace $\mathscr{H}_{d}^{(s)}$. All steps of the simulation procedure are similar to the qutrit simulation procedure for changing inner product explained in the main text.

We now discuss how this simulation procedure for changing the inner product can be used for simulating PT-symmetric dynamics in $d$ dimensions using a $2 d$-dimensional system. Similar to the $d=2$ case discussed in Sec. E, the input to the simulation procedure is $\rho \in \mathcal{B}\left(\mathscr{H}_{d}^{(s)}\right)$ embedded as $\sigma:=$ $\rho \oplus \mathbf{0} \in \mathcal{B}\left(\mathscr{H}_{d}^{(s)} \oplus \mathscr{H}_{d}^{(a)}\right)$ and a time $t>0$. The simulation steps are as follows:

1. The agent calculates $\eta$ satisfying the quasi-Hermiticity condition $H_{\mathrm{PT}}^{\dagger}=\eta H_{\mathrm{PT}} \eta^{-1}$ with $\|\eta\|=1$.

2. The agent implements the procedure described above to simulate $\mathcal{G}_{\eta}$ by setting $\tilde{\eta}=\eta$ and for a single copy of $\sigma$ [Eq. (F1)]. 
3. The agent calculates $h_{\mathrm{PT}}=\eta^{1 / 2} H_{\mathrm{PT}} \eta^{-1 / 2}$ embedded in $\mathcal{B}\left(\mathscr{H}_{d}^{(s)} \oplus \mathscr{H}_{d}^{(a)}\right)$, and implements the unitary operator $e^{-\mathrm{i}\left(h_{\mathrm{PT}} \oplus \mathbf{0}\right) t}$.

4. The agent applies the procedure described above to simulate $\mathcal{G}_{\kappa \eta^{-1}}$, by setting $\tilde{\eta}=\kappa \eta^{-1}$ such that $\kappa=\frac{1}{\left\|\eta^{-1}\right\|}$ [Eq. (F1)].

The output of this procedure is the state $\frac{U_{\mathrm{PT} \rho} U_{\mathrm{PT}}^{\dagger}}{\operatorname{tr}\left(U_{\mathrm{PT}} \rho U_{\mathrm{PT}}^{\dagger}\right)}$ with probability $\frac{1}{\left\|\eta_{2}^{-1}\right\|} \operatorname{tr}\left(U_{\mathrm{PT}} \rho U_{\mathrm{PT}}^{\dagger}\right)$.

\section{APPENDIX G: ADDITIONAL DETAILS ON THE VERIFICATION SCHEME}

We now prove a threshold distance $D_{\text {th }}$ for the tomographic verification scheme, for the qutrit procedure simulating the change in inner product by an arbitrary $\eta$, provided in the main text. The scheme allows a verifier to distinguish an honest prover implementing the operation $\mathcal{G}_{\eta}$ from a dishonest prover failing to implement the same. We assume that the dishonest prover implements only unitary operations, on the qubit subspace, drawn from the set $\left\{U_{j} \oplus \mathbf{1}: U_{j} \in \mathcal{B}\left(\mathscr{H}_{2}\right)\right\}$, where each $U_{j}$ is selected with probability $p_{j}$, and the system is discarded with probability $p:=1-\sum_{j} p_{j}<1$. The quantum operation implemented by the dishonest prover is given by

$$
\hat{\mathcal{G}}_{\eta}(\bullet)=\sum_{j} p_{j}\left(U_{j} \oplus \mathbf{1}\right)^{\dagger} \bullet\left(U_{j} \oplus \mathbf{1}\right) .
$$

We now derive a lower bound for the induced Schatten $(1 \rightarrow$ 1)-norm distance [61] between the inner-product changing operation $\mathcal{G}_{\eta} \oplus \mathbf{0}$ and the implemented operation $\hat{\mathcal{G}}_{\eta}$. Note that

$$
\left\|\mathcal{G}_{\eta} \oplus \mathbf{0}-\hat{\mathcal{G}}_{\eta}\right\|_{1 \rightarrow 1}=\max _{T \in \mathcal{B}\left(\mathscr{H}_{3}\right)} \frac{\left\|\mathcal{G}_{\eta} \oplus \mathbf{1}(T)-\hat{\mathcal{G}}_{\eta}(T)\right\|_{\mathrm{tr}}}{\|T\|_{\mathrm{tr}}},
$$

where $\|T\|_{\text {tr }}=\operatorname{tr}\left(\sqrt{T^{\dagger} T}\right)$. For $T=\frac{1}{3} I_{3},\|T\|_{\text {tr }}=1$. Therefore,

$$
\begin{aligned}
& \left\|\mathcal{G}_{\eta} \oplus \mathbf{0}-\hat{\mathcal{G}}_{\eta}\right\|_{1 \rightarrow 1} \\
& \geqslant \frac{1}{3}\left\|\mathcal{G}_{\eta} \oplus \mathbf{0}\left(I_{3}\right)-\hat{\mathcal{G}}_{\eta}\left(I_{3}\right)\right\|_{\mathrm{tr}} \\
& =\frac{1}{3}\left\|\eta \oplus \mathbf{1}-\sum_{j} p_{j}\left(U_{j} \oplus \mathbf{1}\right)^{\dagger} I_{3}\left(U_{j} \oplus \mathbf{1}\right)\right\|_{\mathrm{tr}} \\
& =\frac{1}{3}\left\|\eta \oplus \mathbf{1}-(1-p) I_{3}\right\|_{\mathrm{tr}} .
\end{aligned}
$$

We assume that the eigenvalues of $\eta$ are denoted by $\lambda_{1}, \lambda_{2}$. The eigenvalues satisfy $1 \geqslant \lambda_{1}>\lambda_{2}>0$ for any nontrivial $\eta$, i.e., $\eta \neq I_{3}$. The trace distance $\left\|\eta \oplus \mathbf{1}-(1-p) I_{3}\right\|_{\text {tr }}=\mid \lambda_{1}-$ $(1-p)|+| \lambda_{2}-(1-p) \mid$. For any $p \in[0,1)$, it can be further verified that $\left\|\eta \oplus \mathbf{1}-(1-p) I_{3}\right\|_{\mathrm{tr}} \geqslant\left(\lambda_{1}-\lambda_{2}\right)$. Therefore,

$$
D_{\text {th }}:=\frac{\left(\lambda_{1}-\lambda_{2}\right)}{3} \leqslant\left\|\mathcal{G}_{\eta} \oplus \mathbf{0}-\hat{\mathcal{G}}_{\eta}\right\|_{1 \rightarrow 1} .
$$

The above given value for $D_{\text {th }}$ allows the verifier to distinguish an honest prover from a dishonest one, provided the honest prover implements the operation $\mathcal{G}_{\eta}$ with error less than $D_{\text {th }}$, where error is quantified by the induced Schatten $(1 \rightarrow 1)$ norm.
[1] J. Sakurai and J. Napolitano, Modern Quantum Mechanics, 3rd ed. (Cambridge University Press, Cambridge, 2007).

[2] C. M. Bender and S. Boettcher, Real Spectra in Non-Hermitian Hamiltonians Having $\mathscr{P} \mathscr{T}$ Symmetry, Phys. Rev. Lett. 80, 5243 (1998).

[3] C. M. Bender, D. C. Brody, and H. F. Jones, Complex Extension of Quantum Mechanics, Phys. Rev. Lett. 89, 270401 (2002).

[4] C. M. Bender, D. C. Brody, and H. F. Jones, Must a Hamiltonian be Hermitian? Am. J. Phys. 71, 1095 (2003).

[5] C. M. Bender, Introduction to $\mathscr{P} \mathscr{T}$-symmetric quantum theory, Contemp. Phys. 46, 277 (2005).

[6] C. E. Rüter, K. G. Makris, R. El-Ganainy, D. N. Christodoulides, M. Segev, and D. Kip, Observation of parity-time symmetry in optics, Nat. Phys. 6, 192 (2010).

[7] J. Schindler, A. Li, M. C. Zheng, F. M. Ellis, and T. Kottos, Experimental study of active LRC circuits with $\mathcal{P} \mathcal{T}$ symmetries, Phys. Rev. A 84, 040101(R) (2011).

[8] S. Bittner, B. Dietz, U. Günther, H. L. Harney, M. Miski-Oglu, A. Richter, and F. Schäfer, $\mathscr{P} \mathscr{T}$ Symmetry and Spontaneous Symmetry Breaking in a Microwave Billiard, Phys. Rev. Lett. 108, 024101 (2012).

[9] B. Peng, Ş. K. Özdemir, F. Lei, F. Monifi, M. Gianfreda, G. L. Long, S. Fan, F. Nori, C. M. Bender, and L. Yang, Parity-time- symmetric whispering-gallery microcavities, Nat. Phys. 10, 394 (2014).

[10] Z. Zhang, Y. Zhang, J. Sheng, L. Yang, M.-A. Miri, D. N. Christodoulides, B. He, Y. Zhang, and M. Xiao, Observation of Parity-Time Symmetry in Optically Induced Atomic Lattices, Phys. Rev. Lett. 117, 123601 (2016).

[11] L. Xiao, X. Zhan, Z. Bian, K. Wang, X. Zhang, X. Wang, J. Li, K. Mochizuki, D. Kim, N. Kawakami et al., Observation of topological edge states in parity-time-symmetric quantum walks, Nat. Phys. 13, 1117 (2017).

[12] R. El-Ganainy, K. G. Makris, M. Khajavikhan, Z. H. Musslimani, S. Rotter, and D. N. Christodoulides, NonHermitian physics and PT symmetry, Nat. Phys. 14, 11 (2018).

[13] Y. Wu, W. Liu, J. Geng, X. Song, X. Ye, C.-K. Duan, X. Rong, and J. Du, Observation of parity-time symmetry breaking in a single-spin system, Science 364, 878 (2019).

[14] J. Zhang, L. Li, G. Wang, X. Feng, B.-O. Guan, and J. Yao, Parity-time symmetry in wavelength space within a single spatial resonator, Nat. Commun. 11, 3217 (2020).

[15] A. Mostafazadeh, Exact $P T$-symmetry is equivalent to Hermiticity, J. Phys. A 36, 7081 (2003).

[16] K. G. Makris, R. El-Ganainy, D. N. Christodoulides, and Z. H. Musslimani, Beam Dynamics in $\mathcal{P} \mathcal{T}$ Sym- 
metric Optical Lattices, Phys. Rev. Lett. 100, 103904 (2008).

[17] C.-Y. Ju, A. Miranowicz, G.-Y. Chen, and F. Nori, NonHermitian Hamiltonians and no-go theorems in quantum information, Phys. Rev. A 100, 062118 (2019).

[18] S. Croke, $\mathcal{P} \mathcal{T}$-symmetric Hamiltonians and their application in quantum information, Phys. Rev. A 91, 052113 (2015).

[19] C. M. Bender, D. C. Brody, J. Caldeira, U. Günther, B. K. Meister, and B. F. Samsonov, PT-symmetric quantum state discrimination, Philos. Trans. R. Soc. A 371, 20120160 (2013).

[20] X. Zhan, K. Wang, L. Xiao, Z. Bian, Y. Zhang, B. C. Sanders, C. Zhang, and P. Xue, Experimental quantum cloning in a pseudounitary system, Phys. Rev. A 101, 010302(R) (2020).

[21] C. M. Bender, D. C. Brody, H. F. Jones, and B. K. Meister, Faster Than Hermitian Quantum Mechanics, Phys. Rev. Lett. 98, 040403 (2007).

[22] A. Mostafazadeh, Hamiltonians generating optimal-speed evolutions, Phys. Rev. A 79, 014101 (2009).

[23] A. K. Pati, Violation of invariance of entanglement under local PT symmetric unitary, arXiv:1404.6166.

[24] S.-L. Chen, G.-Y. Chen, and Y.-N. Chen, Increase of entanglement by local $\mathcal{P} \mathcal{T}$-symmetric operations, Phys. Rev. A 90, 054301 (2014).

[25] Y.-C. Lee, M.-H. Hsieh, S. T. Flammia, and R.-K. Lee, Local $\mathcal{P} \mathcal{T}$ Symmetry Violates The No-Signaling Principle, Phys. Rev. Lett. 112, 130404 (2014).

[26] F. G. Scholtz, H. B. Geyer, and F. J. W. Hahne, Quasi-Hermitian operators in quantum mechanics and the variational principle, Ann. Phys. (NY) 213, 74 (1992).

[27] A. Mostafazadeh, Conceptual aspects of $\mathcal{P} \mathcal{T}$-symmetry and pseudo-Hermiticity: A status report, Phys. Scr. 82, 038110 (2010).

[28] A. Mostafazadeh, Pseudo-Hermitian representation of quantum mechanics, Int. J. Geom. Meth. Mod. Phys. 7, 1191 (2010).

[29] M. Znojil, Non-self-adjoint Operators in Quantum Physics: Ideas, People, and Trends, edited by F. Bagarello, J. P. Gazeau, F. H. Szafraniec, and M. Znojil (Wiley, New York, 2015).

[30] A. Mostafazadeh, Energy observable for a quantum system with a dynamical Hilbert space and a global geometric extension of quantum theory, Phys. Rev. D 98, 046022 (2018).

[31] D.-J. Zhang, Q.-h. Wang, and J. Gong, Time-dependent $\mathscr{P} \mathscr{T}$-symmetric quantum mechanics in generic non-Hermitian systems, Phys. Rev. A 100, 062121 (2019).

[32] D. C. Brody, Consistency of PT-symmetric quantum mechanics, J. Phys. A 49, 10LT03 (2016).

[33] Z.-P. Liu, J. Zhang, Ş. K. Özdemir, B. Peng, H. Jing, X.-Y. Lü, C.-W. Li, L. Yang, F. Nori, and Y.-x. Liu, Metrology with $\mathcal{P} \mathcal{T}$ Symmetric Cavities: Enhanced Sensitivity Near the $\mathcal{P} \mathcal{T}$-Phase Transition, Phys. Rev. Lett. 117, 110802 (2016).

[34] W. Chen, Ş. K. Özdemir, G. Zhao, J. Wiersig, and L. Yang, Exceptional points enhance sensing in an optical microcavity, Nature (London) 548, 192 (2017).

[35] H. Hodaei, A. U. Hassan, S. Wittek, H. Garcia-Gracia, R. El-Ganainy, D. N. Christodoulides, and M. Khajavikhan, Enhanced sensitivity at higher-order exceptional points, Nature (London) 548, 187 (2017).

[36] X. Zhu, L. Feng, P. Zhang, X. Yin, and X. Zhang, One-way invisible cloak using parity-time symmetric transformation optics, Opt. Lett. 38, 2821 (2013).
[37] D. L. Sounas, R. Fleury, and A. Alù, Unidirectional cloaking based on metasurfaces with balanced loss and gain, Phys. Rev. Applied 4, 014005 (2015).

[38] H. Ramezani, T. Kottos, R. El-Ganainy, and D. N Christodoulides, Unidirectional nonlinear PT-symmetric optical structures, Phys. Rev. A 82, 043803 (2010).

[39] J. Li, A. K. Harter, J. Liu, L. de Melo, Y. N. Joglekar, and L. Luo, Observation of parity-time symmetry breaking transitions in a dissipative Floquet system of ultracold atoms, Nat. Commun. 10, 855 (2019).

[40] L. Xiao, X. Qiu, K. Wang, B. C. Sanders, W. Yi, and P. Xue, Topology with broken parity-time symmetry, arXiv:1906.07468.

[41] U. Günther and B. F. Samsonov, $\mathscr{P} \mathscr{T}$-symmetric brachistochrone problem, Lorentz boosts, and nonunitary operator equivalence classes, Phys. Rev. A 78, 042115 (2008).

[42] U. Günther and B. F. Samsonov, Naimark-Dilated $\mathscr{P} \mathscr{T}$ Symmetric Brachistochrone, Phys. Rev. Lett. 101, 230404 (2008).

[43] C. Zheng, L. Hao, and G. L. Long, Observation of a fast evolution in a parity-time-symmetric system, Phil. Trans. R. Soc. A. 371, 20120053 (2013).

[44] J.-S. Tang, Y.-T. Wang, S. Yu, D.-Y. He, J.-S. Xu, B.-H. Liu, G. Chen, Y.-N. Sun, K. Sun, Y.-J. Han et al., Experimental investigation of the no-signalling principle in parity-time symmetric theory using an open quantum system, Nat. Photonics 10, 642 (2016).

[45] K. Kawabata, Y. Ashida, and M. Ueda, Information Retrieval and Criticality in Parity-Time-Symmetric Systems, Phys. Rev. Lett. 119, 190401 (2017).

[46] M. Huang, A. Kumar, and J. Wu, Embedding, simulation and consistency of $\mathscr{P} \mathscr{T}$-symmetric quantum theory, Phys. Lett. A 382, 2578 (2018).

[47] L. Xiao, K. Wang, X. Zhan, Z. Bian, K. Kawabata, M. Ueda, W. Yi, and P. Xue, Observation of Critical Phenomena in ParityTime-Symmetric Quantum Dynamics, Phys. Rev. Lett. 123, 230401 (2019).

[48] W.-C. Gao, C. Zheng, L. Liu, T.-J. Wang, and C. Wang, Experimental simulation of the parity-time symmetric dynamics using photonic qubits, Opt. Express 29, 517 (2021).

[49] F. Strocchi, An Introduction to the Mathematical Structure of Quantum Mechanics: A Short Course for Mathematicians, 2nd ed., Advanced Series in Mathematical Physics (World Scientific, Singapore, 2008).

[50] I. Gelfand and M. Neumark, On the imbedding of normed rings into the ring of operators in Hilbert space, Rec. Math. [Mat. Sbornik] N.S. 12, 197 (1943).

[51] I. Segal, Irreducible representations of operator algebras, Bull. Amer. Math. Soc. 53, 73 (1947).

[52] S. P. Gudder, Axiomatic operational quantum mechanics, Rep. Math. Phys. 16, 147 (1979).

[53] We remark that supernormalized functionals are valid states in some other frameworks for describing system evolution under non-Hermitian Hamiltonians [66-68].

[54] The map lift is defined to act only on the normalized and subnormalized vectors in $\mathscr{H}$. The domain of lift in Fig. 1(c) is shown to be $\mathscr{H}$ for simplicity, but is specified rigorously in Appendix A3.

[55] J. Conway, A Course in Functional Analysis, 2nd ed. (SpringerVerlag, New York, 2007). 
[56] I. L. Chuang and M. A. Nielsen, Prescription for experimental determination of the dynamics of a quantum black box, J. Mod. Opt. 44, 2455 (1997).

[57] I. Bongioanni, L. Sansoni, F. Sciarrino, G. Vallone, and P. Mataloni, Experimental quantum process tomography of nontrace-preserving maps, Phys. Rev. A 82, 042307 (2010).

[58] A. Mostafazadeh, Pseudo-Hermiticity versus PT-symmetry III: Equivalence of pseudo-Hermiticity and the presence of antilinear symmetries, J. Math. Phys. 43, 3944 (2002).

[59] The ratio of nonzero measurement outcomes to the total number of copies approaches the success probability by the law of large numbers [69]. Our setting assumes that multiple copies of the state $\sigma$ are provided to the agent by an external agent who has the knowledge of $\sigma$, and not prepared by the agent implementing the inner-product changing channel, say, by cloning.

[60] W. F. Stinespring, Positive functions on $C^{*}$-algebras, Proc. Am. Math. Soc. 6, 211 (1955).

[61] V. Paulsen, Completely Bounded Maps and Operator Algebras, Cambridge Studies in Advanced Mathematics (Cambridge University Press, Cambridge, 2003).

[62] T. Sugiyama, P. S. Turner, and M. Murao, Error probability analysis in quantum tomography: A tool for evaluating experiments, Phys. Rev. A 83, 012105 (2011).
[63] A. M. Steinberg, P. G. Kwiat, and R. Y. Chiao, Measurement of the Single-Photon Tunneling Time, Phys. Rev. Lett. 71, 708 (1993).

[64] A. Morvan, V. V. Ramasesh, M. S. Blok, J. M. Kreikebaum, K. O’Brien, L. Chen, B. K. Mitchell, R. K. Naik, D. I. Santiago, and I. Siddiqi, Qutrit Randomized Benchmarking, Phys. Rev. Lett. 126, 210504 (2021).

[65] M. Kononenko, M. A. Yurtalan, J. Shi, and A. Lupascu, Characterization of control in a superconducting qutrit using randomized benchmarking, Phys. Rev. Research 3, L042007 (2021).

[66] A. Mostafazadeh, Quantum Brachistochrone Problem and the Geometry of the State Space in Pseudo-Hermitian Quantum Mechanics, Phys. Rev. Lett. 99, 130502 (2007).

[67] E.-M. Graefe, H. J. Korsch, and A. E. Niederle, Quantumclassical correspondence for a non-Hermitian Bose-Hubbard dimer, Phys. Rev. A 82, 013629 (2010).

[68] R. Uzdin, U. Günther, S. Rahav, and N. Moiseyev, Timedependent Hamiltonians with $100 \%$ evolution speed efficiency, J. Phys. A 45, 415304 (2012).

[69] F. M. Dekking, C. Kraaikamp, H. P. Lopuhaä, and L. E. Meester, A Modern Introduction to Probability and Statistics: Understanding Why and How (Springer-Verlag, London, 2005). 